\title{
Précipitation de carbonates de cations divalents dans les systèmes lacustres : intérêt, état des connaissances des mécanismes et suggestions (Revue critique de la littérature) \\ Carbonate precipitation of divalent cations in lakes: interest, current knowledge of the mechanisms and suggestions (a critical review of the literature)
}

\section{Théophile Kamgaing}

Volume 28, numéro 2, 2015

Reçu le 15 avril 2013, accepté le 13 août 2014

URI : https://id.erudit.org/iderudit/1032292ar

DOI : https://doi.org/10.7202/1032292ar

Aller au sommaire du numéro

Éditeur(s)

Université du Québec - INRS-Eau, Terre et Environnement (INRS-ETE)

ISSN

1718-8598 (numérique)

Découvrir la revue

Citer cet article

Kamgaing, T. (2015). Précipitation de carbonates de cations divalents dans les systèmes lacustres : intérêt, état des connaissances des mécanismes et suggestions (Revue critique de la littérature). Revue des sciences de l'eau / Journal of Water Science, 28(2), 81-102. https://doi.org/10.7202/1032292ar

\section{Résumé de l'article}

Les carbonates de fer, de manganèse, de calcium et de magnésium précipités en solution des lacs sont documentés. Indicateurs de climat et de variation des caractéristiques chimiques des lacs, ils sont de plus en plus recherchés dans les sédiments. Souvent incomplets, les mécanismes de leur précipitation dans la colonne d'eau du lac et dans les eaux interstitielles sont reprécisés dans cette étude. D’après la littérature, la sidérite et la rhodochrosite précipitent en milieu réducteur saturé de carbonate de fer et de carbonate de manganèse respectivement. Ces prévisions sont confirmées dans cette étude, le potentiel redox étant un paramètre déterminant pour la précipitation du carbonate de fer. Toutefois, la littérature ne décrit pas suffisamment l'origine (géochimique ou biologique) des éléments constitutifs des solides carbonatés lacustres, encore moins les phénomènes qui stabilisent ces derniers ou les rendent vulnérables (dissolution). Cette étude apporte plus de précisions à l'endogènèse de ces carbonates, l'origine de leurs éléments constitutifs étant prise en compte. Elle montre que le caractère de l'eau (agressif ou incrustant) pourrait avoir un impact considérable sur le devenir de ces carbonates. Ainsi un dégazage forcé d'un lac rendrait ses eaux incrustantes, caractère idéal pour la précipitation des carbonates, mais néfaste à la stabilité des strates qui changent de composition après précipitation d'espèces chimiques. Par conséquent, tout projet de dégazage de lac devrait prendre en considération ce paramètre (caractère de l'eau) dans sa conception, sa mise en oeuvre et son exploitation. 


\section{PRÉCIPITATION DE CARBONATES DE CATIONS DIVALENTS DANS LES SYSTÈMES LACUSTRES: INTÉRÊT, ÉTAT DES CONNAISSANCES DES MÉCANISMES ET SUGGESTIONS (REVUE CRITIQUE DE LA LITTÉRATURE)}

Carbonate precipitation of divalent cations in lakes: interest, current knowledge of the mechanisms and suggestions (a critical review of the literature)

THÉOPHILE KAMGAING

Laboratoire LACHINGE (Chimie des Nuisances et Génie de l'Environnement), Université de Dschang, B.P. 67, Dschang, Cameroun

Reçu le 15 avril 2013, accepté le 13 août 2014

\section{RÉSUMÉ}

Les carbonates de fer, de manganèse, de calcium et de magnésium précipités en solution des lacs sont documentés. Indicateurs de climat et de variation des caractéristiques chimiques des lacs, ils sont de plus en plus recherchés dans les sédiments. Souvent incomplets, les mécanismes de leur précipitation dans la colonne d'eau du lac et dans les eaux interstitielles sont reprécisés dans cette étude. D'après la littérature, la sidérite et la rhodochrosite précipitent en milieu réducteur saturé de carbonate de fer et de carbonate de manganèse respectivement. Ces prévisions sont confirmées dans cette étude, le potentiel redox étant un paramètre déterminant pour la précipitation $\mathrm{du}$ carbonate de fer. Toutefois, la littérature ne décrit pas suffisamment l'origine (géochimique ou biologique) des éléments constitutifs des solides carbonatés lacustres, encore moins les phénomènes qui stabilisent ces derniers ou les rendent vulnérables (dissolution). Cette étude apporte plus de précisions à l'endogènèse de ces carbonates, l'origine de leurs éléments constitutifs étant prise en compte. Elle montre que le caractère de l'eau (agressif ou incrustant) pourrait avoir un impact considérable sur le devenir de ces carbonates. Ainsi un dégazage forcé d'un lac rendrait ses eaux incrustantes, caractère idéal pour la précipitation des carbonates, mais néfaste à la stabilité des strates qui changent de composition après précipitation d'espèces chimiques. Par conséquent, tout projet de dégazage de lac devrait prendre en considération ce paramètre (caractère de l'eau) dans sa conception, sa mise en œuvre et son exploitation.

Mots-clés : Lac, colonne d'eau, solides carbonatés, éléments constitutifs, indicateurs climatiques, mécanismes.

\section{ABSTRACT}

Carbonates that precipitate in the water column of the lakes are documented: these include siderite, rhodochrosite, calcite, magnesite, and dolomite. As climate indicators and 
indicators of changes in chemical characteristics of lakes, they are increasingly monitored in sediments. Often incomplete, the mechanisms of their precipitation in the water column of the lake and in pore waters are reviewed in this study. According to the literature, siderite and rhodochrosite precipitate in reducing environments saturated with iron carbonate and manganese carbonate respectively. These predictions are confirmed in this study, the redox potential being a key parameter for the precipitation of iron carbonate. However, the literature does not sufficiently describe the origin (geochemical or biological) of lacustrine solid carbonate components or the phenomena that stabilize or make them vulnerable (dissolution). This study provides further details on the endogenesis of these carbonates by taking account of the origin of their components. This study also shows that the character of the water (aggressive or encrusting) could have a significant impact on the fate of these carbonates. Thus, degassing a lake might make water encrusting; this encrusting character of water is ideal for the precipitation of carbonates, but detrimental to the stability of layers whose composition changes after precipitation of chemical species. Therefore any lake degassing project should consider this parameter (aggressive or encrusting nature of the water) in its design, implementation and operation.

\section{Keywords: Lake, water column, solid carbonates, compo- nents, climate indicators, mechanisms.}

\section{INTRODUCTION}

Les carbonates sont les minéraux les plus fréquents qui précipitent dans des environnements riches en méthane (LEIN, 2004). Plusieurs facteurs sont favorables à leur précipitation, entre autres l'évaporation et le dégazage, deux phénomènes qui modifient considérablement la composition de la solution des lacs. Alors que le réchauffement du climat provoque l'évaporation, le retournement des eaux rompt l'équilibre des strates et provoque le dégazage. Dans les deux cas, la concentration des espèces dissoutes change et certaines parviennent à saturation (EUGSTER et HARDIE, 1978; PIENITZ et al., 1992; ROCA et JULIA, 1997). En effet, au fur et à mesure que le lac s'évapore, le coefficient de saturation des espèces minérales augmente progressivement jusqu'à l'optimum où carbonates sulfates et chlorures précipitent (RODO et al., 2002).Nous nous intéressons à la précipitation endogène de carbonates de calcium, de fer, de manganèse et de magnésium qui sont des indicateurs climatiques indéniables (MASON et al.,1991; SHAPLEY et al., 2002; TELLER et LAST, 1990). Plusieurs auteurs ont décrit les mécanismes de précipitation de ces carbonates sans tenir compte, si oui partiellement, de l'origine de leurs éléments constitutifs (STABEL, 1986; RAJAN et al., 1996; DAG HONGVE, 1997; DEAN, 2009; JIMENEZ-LOPEZ et ROMANEK, 2004; KONOVALOV et al., 2004; KRYLOV et al., 2008; DEAN et DONER, 2011).

Pourtant, l'implication des algues dans la nucléation de la calcite en solution des lacs (STABEL, 1986), la précipitation endogène de la sidérite tantôt à partir du fer biogénique et du bicarbonate géochimique (TEUTSCH et al., 2009), tantôt à partir du bicarbonate biogénique (KRYLOV et al., 2008) devraient inciter à tenir compte des origines des constituants des solides carbonatés lacustres dans les mécanismes de leur précipitation.

Après une revue profonde de la littérature sur le sujet, nous proposons dans cette étude trois origines des éléments constitutifs des solides carbonatés qui précipitent en solution dans les lacs : origine géochimique biologique ou biogéochimique, selon la nature des anions et des cations de ces solides. La prise en compte de ces origines apporterait plus de précisions à l'endogenèse des phases solides carbonatées des sédiments lacustres. Ainsi, au lieu de parler de la sidérite endogénique, terme qui ne précise pas l'origine des constituants de la sidérite, il conviendrait de parler de la sidérite endogéochimique quand les deux éléments qui la constituent sont d'origine géochimique, et de la sidérite endo-biogéochimique quand l'un des éléments constitutifs a une origine biologique et l'autre géochimique.

Par ailleurs, si la littérature abonde sur les mécanismes de décomposition des oxydes et hydroxydes en milieu marin et lacustre, lesquels mécanismes ne font pas l'unanimité, elle ne décrit pas suffisamment les phénomènes qui décomposent ou stabilisent les carbonates solides, phénomènes qui permettraient de comprendre pourquoi certains sédiments lacustres sont plus riches en carbonates que d'autres de composition voisine, voire identique.

Cette étude tente d'harmoniser les données de la littérature tout en apportant des éléments de réponse aux préoccupations évoquées.

\section{INTÉRÊT DE L'ÉTUDE}

Le manganèse et le fer rencontrés dans les sédiments des lacs sont tantôt sous forme d'oxydes et d'hydroxydes (WALTER et LISA. 2011) tantôt sous forme de carbonates (BAHRIG, 1985; CALLENDER et GRANINA, 1992; EMERSON, 1976; KRYLOV et al., 2008; LIM et al., 2004; POSTMA, 1982). La sidérite, une des formes carbonatées du fer est retrouvée dans les sédiments des lacs stratifiés et profonds ayant un potentiel redox négatif et très pourvus en fer(II) et en hydrogénocarbonate (DAVISON, 1993; RAJAN et al., 1996). L'environnement dans lequel se forme la sidérite lacustre semble donc particulier. On cherche à comprendre le mécanisme par lequel elle précipite, les conséquences de sa précipitation sur la stabilité du lac (risque 
de dégazage consécutif à une rupture d'équilibre des strates), l'effet du climat sur sa précipitation afin d'établir un lien de causalité s'il en existe. En effet, les principes géochimiques et les observations de terrain montrent que les lacs alcalins qui subissent l'évaporation peuvent devenir des réacteurs idéaux de carbonate de calcium (DEAN, 2009; SHAPLEY et al., 2002). Les anomalies du climat peuvent donc influencer l'endogénèse des carbonates solides. Il en est de même du dégazage d'un lac, qu'il soit spontané ou provoqué. BERNARD et SIMONE (1987), analysant les sédiments superficiels du Lac Nyos après le dégazage spontané de 1986, lequel fit 1700 victimes (KLING et al., 1987) signalent la présence de la sidérite précipitée dans la colonne d'eau de ce lac. Une analyse profonde des sédiments inférieurs pourrait révéler des intervalles carbonatés témoins des évasions antérieures de gaz, la même cause produisant le même effet. Il apparait ainsi que les carbonates solides précipités en solution des lacs pourraient être des indicateurs climatiques, mais aussi un paramètre de suivi des milieux lacustres.

\section{MATÉRIELS ET MÉTHODES}

Chaque solide carbonaté pris séparément est étudié selon la séquence suivante : revue de la littérature et harmonisation des mécanismes de formation en tenant compte de l'origine des éléments constitutifs du solide - compléments de mécanismes études expérimentales de la précipitation des solides carbonatés au moyen du diagramme potentiel redox-pH : les expériences visent à confirmer ou infirmer certaines données de la littérature à partir des considérations simples de la thermodynamique et des procédures expérimentales simplifiées; les constantes thermodynamiques des équilibres énumérés dans cette étude peuvent être consultées dans les compilations critiques de l'Union Internationale de Chimie Pure et Appliquée (IUPAC) sur les carbonates des systèmes aquatiques. Les analyses physicochimiques sont prises en considération et doivent corréler les analyses radiocristallographiques et microscopiques. L'analyse des phénomènes qui fragilisent ou stabilisent les solides carbonatés ainsi que l'influence de l'évaporation et du dégazage spontané ou forcé sur l'endogénèse des carbonates solides seront abordés en dernier.

\subsection{Diagramme potentiel $-\mathrm{pH}$ des systèmes $\left(\mathrm{Fe}, \mathrm{CO}_{2}, \mathrm{H}_{2} \mathrm{O}\right)$ $\left(\mathrm{Mn}, \mathrm{CO}_{2}, \mathrm{H}_{2} \mathrm{O}\right)$ et $\left(\mathrm{Ca}, \mathrm{CO}_{2}, \mathrm{H}_{2} \mathrm{O}\right)$}

Ces diagrammes sont connus sous l'appellation " diagrammes de POURBAIX (1963) » qu'il conviendrait d'établir en tenant compte des équilibres chimiques possibles en solution des lacs. Rappelons tout de même qu'il s'agit de représentations graphiques d'un ensemble d'informations relatives aux équilibres qui peuvent exister en milieu aqueux entre des espèces chimiques comportant un même élément à différents degrés d'oxydation.
Pour établir les diagrammes d'équilibre, nous avons retenu les concentrations suivantes des cations métalliques dissous et des espèces carboniques totales $\mathrm{CO}_{2 \mathrm{~T}}$

- fer et manganèse dissous : $10^{-5} \mathrm{~mol} / \mathrm{l}$ et $10^{-3} \mathrm{~mol} / \mathrm{l}$

- $\mathrm{CO}_{2}$ total: $10^{-2} \mathrm{~mol} / \mathrm{l}$ (acide carbonique + hydrogénocarbonate + carbonate)

Il est à remarquer d'une part que deux concentrations sont attribuées aux espèces métalliques. En effet, l'hypolimnion des lacs stratifiés est généralement plus concentré en espèces dissoutes que l'épilimnion; d'autre part, les quantités considérées dans cette étude sont sous-estimées quand on sait que certains lacs contiennent d'avantage du $\mathrm{CO}_{2}$ dissous pour ne parler que de cette entité $\left(340 \mathrm{mmol} / \mathrm{kg} \mathrm{CO}_{2}\right.$ à $206 \mathrm{~m}$ de profondeur du lac Nyos (KUSAKABE et al., 2000) ; Néanmoins ces quantités sous-estimées suffisent pour déterminer de façon précise les domaines de potentiel redox et de $\mathrm{pH}$ favorables à la précipitation de solides carbonatés.

\subsection{Expérimentation}

En ajustant le pH et le potentiel redox d'une eau synthétique à des valeurs convenables déduites des diagrammes établis, il est possible par simple agitation moyennant quelques précautions de précipiter des solides carbonatés.

\subsubsection{Procédure expérimentale}

La solubilité de l'hydroxyde de calcium est limitée. Par contre si on fait barboter du dioxyde de carbone dans de l'eau contenant cet électrolyte faible, on obtient une solution de calcium hydrogénocarbonate $\mathrm{Ca}\left(\mathrm{HCO}_{3}\right)_{2}$. Après filtration, le perméat est introduit dans un réacteur, désaéré à l'azote et maintenu à $25^{\circ} \mathrm{C}$ sous agitation magnétique dans un bain thermorégulé. Ce filtrat a un caractère oxydant (potentiel redox > 0) même après barbotage de l'azote. Pour lui conférer un caractère réducteur lorsque c'est nécessaire, le dithionite de sodium $\mathrm{Na}_{2} \mathrm{~S}_{2} \mathrm{O}_{4}$ (MAYHEW, 1978) est ajouté à la solution. Parmi les réducteurs testés, ce dernier a donné satisfaction, baissant suffisamment le potentiel redox du milieu à des valeurs convenables. Selon les cas, le sulfate ferreux est ajouté dans le but de précipiter la sidérite ou alors du chlorure de manganèse pour précipiter la rhodochrosite. Par addition de soude, on élève le $\mathrm{pH}$ du milieu pour provoquer la précipitation ; la valeur du $\mathrm{pH}$ ne doit pas dépasser celle que recommande le diagramme établi, au risque de former plutôt des oxydes et hydroxydes. Au bout de $24 \mathrm{~h}$, l'équilibre est sûrement atteint.

\subsubsection{Méthode d'analyse chimique}

Une analyse est faite avant et après précipitation et concerne : 
- le dosage de $\mathrm{Ca}^{2+}, \mathrm{Fe}^{2+}$ ou $\mathrm{Mn}^{2+}$

- le dosage de $\mathrm{CO}_{2}$ total

- la mesure du pH

- la mesure du potentiel redox

Les ions $\mathrm{Ca}^{2+}, \mathrm{Fe}^{2+}$ et $\mathrm{Mn}^{2+}$ sont dosés à l'aide d'un spectrophotomètre d'absorption atomique (Varian Spectra 10) utilisé en flamme air - acétylène à la longueur d'onde $422,7 \mathrm{~nm}$; $248,3 \mathrm{~nm} ; 279,5 \mathrm{~nm}$ respectivement.

Le $\mathrm{CO}_{2}$ total est mesuré au moyen d'un analyseur de carbone (Dohrman carbon analyser, modèle DC 80). Étantdonné que la précipitation d'une mole de carbonate entraîne la diminution d'une mole de $\mathrm{CO}_{2}$ total et d'une mole de cations considérés, il est possible après analyse chimique de savoir si le précipité est sous forme d'oxyde ou de carbonate.

Le $\mathrm{pH}$ est déterminé au moyen d'un ionomètre à microprocesseur "Orion 901 " et d'une électrode de verre combinée type Ross.

Le potentiel redox est mesuré à l'aide d'une électrode de platine et d'une électrode au calomel saturé.

\subsubsection{Analyse radiocristallographique}

La diffraction des rayons $\mathrm{x}$ des précipités a été réalisée avec un générateur PHILIPS PW 1009 muni d'un tube à anticathode de fer et de chambres à enregistrement photographique. L'identification des phases a été faite par comparaison avec les données contenues dans le fichier publié par le " Joint Committee on Powder Diffraction Standards, J.C.P.D.S». Les paramètres de maille (a et $\mathrm{c}$ ) des précipités du fer ont été calculés en considérant les six réflexions (indices de Miller) les plus fortes de la sidérite $(102,104,110,113,202,116)$.

\subsubsection{Analyse SEM (microscopie électronique à balayage)}

La morphologie des cristaux a été mise en évidence au moyen d'un microscope électronique à balayage à effet de champ, de marque JEOL JSM 6301F.

\section{RÉSULTATS ET DISCUSSION}

\subsection{Mécanisme de formation endogène de la sidérite lacustre $\mathrm{FeCO}_{3}$}

Avant d'aborder un tel mécanisme, il conviendrait de s'interroger sur les origines des constituants du solide carbonaté $\mathrm{FeCO}_{3}$.
4.1.1 Origines de l'ion $\mathrm{Fe}^{2+}$, élément constitutif de $\mathrm{FeCO}_{3}$

4.1.1.1 Source géochimique de l'ion $\mathrm{Fe}^{2++-}$

L'origine géochimique du fer peut être lointaine ; il peut provenir d'un aquifere profond qui recouvre un substratum rocheux du crétacé (DEAN et DONER, 2011). Les sources volcaniques du fer ne sont pas à négliger (GLENN, 2008) ; Les oxysols sont également une source importante de fer. Le lessivage de ces sols riches en $\mathrm{Fe}_{2} \mathrm{O}_{3}$ est à l'origine d'importantes quantités de fer rencontrées dans certains lacs de cratère (KAMGAING, 2003; TEUTSCH et al., 2009). Lacidité du milieu accélère le processus selon la réaction

$$
\mathrm{Fe}_{2} \mathrm{O}_{3}+4 \mathrm{H}^{+} \rightarrow 2 \mathrm{Fe}^{2+}+2 \mathrm{H}_{2} \mathrm{O}+\frac{1}{2} \mathrm{O}_{2}
$$

Le fer(II) n'est pas stable en milieu oxygéné. Il est concentré dans les zones anoxiques des lacs stratifiés (DAG HONGVE, 1997). Dans les zones oxiques, il s'oxyde en $\mathrm{Fe}^{3+}$, puis précipite sous forme d'hydroxyde $\mathrm{Fe}(\mathrm{OH})_{3}$. Il convient de rappeler qu'au niveau de l'oxycline, des bactéries acidophiles oxydatives du fer peuvent conduire également au précipité $\mathrm{Fe}(\mathrm{OH})_{3}$ (GLENN, 2008). Lorsque ce dernier atteint les zones anoxiques, il est réduit en fer(II) par des bactéries réductrices du fer selon un mécanisme que nous proposons au paragraphe 2.1.1.2.2. Le temps de résidence de $\mathrm{Fe}^{2+}$ en zone anoxique pouvant être plus court que celui de l'eau, $\mathrm{Fe}^{2+}$ migre alors vers la frontière oxiqueanoxique où il est de nouveau oxydé, et le cycle recommence (DAG HONGVE, 1997; KONOLOV et al., 2004).

\subsubsection{Origine biogénique de l'ion $\mathrm{Fe}^{2+}$ \\ 4.1.1.2.1 Mécanismes de réduction bactérienne de $\mathrm{Fe}(\mathrm{OH})_{3}$ en $\mathrm{Fe}^{2+}$}

L'oxydation bactérienne de $\mathrm{Fe}^{2+}$ en $\mathrm{Fe}(\mathrm{OH})_{3}$ est bien décrite (EHRENREICH et WIDDEL, 1994; STRAUB et al.,1996; STRAUB et BUCHHOLZ-CLEVEN, 1998; WIDDEL et al., 1993). Par contre, les mécanismes de la réduction bactérienne de l'hydroxyde de fer suscitent encore des interrogations. COLEMAN et al. (1993) remarquaient déjà que la sidérite se forme après réduction des oxydes ferriques en ion ferreux par les bactéries sulfatoréductrices Desulfovibrio desulfuricans. GLENN (2008), abondant dans le même sens, signale certains types de bactéries non acidophiles capables d'accumuler l'hydroxydes de fer et donc le fer(III) dans leurs parois cellulaires pendant la nucléation de la sidérite : liptothrax et crenothrix, deux bactéries non acidophiles porteuses de masses floculantes de $\mathrm{Fe}(\mathrm{OH})_{3}$ ont ainsi été identifiées par GLENN dans la rivière Verte "Green river " provenant du lac Gosiute (Colorado). Toutefois, cet auteur n'en dit pas plus sur les mécanismes qui gouvernent la réduction bactérienne de l'hydroxyde de fer. À notre sens, la production d'énergie est au cour de ce processus biologique. En effet, l'oxydation microbiologique des substances chimiques s'accompagne en général de la production d'énergie indispensable à la croissance 
bactérienne. Par conséquent, il n'est pas exclu que la réduction bactérienne de $\mathrm{Fe}(\mathrm{OH})_{3}$ soit nécessaire à la synthèse continue de l'adénosine triphosphate (ATP). Les métabolites de cette synthèse pourraient avoir un rôle important dans la réduction de l'hydroxyde en question. Si tel est le cas, le mécanisme de transfert de ces métabolites est ci-dessous suggéré.

\subsection{Suggestion de mécanismes}

$\mathrm{Fe}(\mathrm{OH})_{3}$ peut être réduit en $\mathrm{Fe}^{2+}$ parce qu'il est un accepteur d'électrons(oxydant). Les électrons proviennent d'un donneur qui est un réducteur. Si le réducteur est la matière organique $\mathrm{MO}$, sa dégradation génère des métabolites, des protons et des électrons. Cette dégradation se fait en général avec production d'ATP, source d'énergie indispensable à la croissance bactérienne (KAPPLER et STRAUB, 2005; LOVLEY, 1993 et 2004; NEALSON et SAFFARINI, 1994; STUCKI, 2006). Protons et électrons sont transmis à l'hydroxyde par l'intermédiaire d'une enzyme bactérienne, la ferriréductase selon le mécanisme ci-après :

$$
\begin{gathered}
\mathrm{MO} \rightarrow \mathrm{e}+\mathrm{H}^{+}+\text {métabolites } \\
\mathrm{ADP}+\mathrm{Pi} \rightarrow \text { ATP } \\
(\mathrm{OH})_{2}-\mathrm{Fe}-\mathrm{OH}+\mathrm{e}+3 \mathrm{H}^{+} \text {ferriréductase } \\
\rightarrow \mathrm{Fe}^{2+}+3 \mathrm{H}_{2} \mathrm{O}
\end{gathered}
$$

Il convient de signaler que dans ce mécanisme la synthèse de l'ATP est couplée à l'oxydation de la matière organique. Le transfert des électrons et des protons à l'hydroxyde est une étape déterminante. Ce transfert est assuré par le contact entre l'enzyme bactérienne et l'hydroxyde.

\subsection{Contact " cellule bactérienne - hydroxyde de fer "}

Le transfert des électrons et des protons dépend de l'efficacité des contacts entre l'hydroxyde et la bactérie (NEVIN et LOVLEY, 2002). MUNCH et OTTOW (2010) étudiant le mécanisme de réduction bactérienne de l'oxyde de fer dans le sol ont montré que le contact optimal s'obtient avec l'oxyde amorphe, non cristallisé. En effet, l'énergie d'activation bactérienne est faible lorsque le contenu énergétique de la substance à activer est élevé ; or, les substances cristallisées ont en général un contenu énergétique faible comparé aux substances amorphes. Par ailleurs, l'hydroxyde colloïdal parce que porteur de charges négatives, repousserait les électrons, rendant le contact difficile (MUNCH et OTTOW, 2010). Le mécanisme de réduction bactérienne de l'hydroxyde de fer dans les eaux interstitielles des lacs serait similaire à celui des oxydes de fer dans le sol. Il s'agit d'une réduction enzymatique qui nécessite un contact direct oxydes/hydroxydes - bactéries pour que le proton et les électrons issus du métabolisme soient transmis aux oxydes ou hydroxydes accepteurs. Certains auteurs ont expérimenté la réduction bactérienne de Fe(III) à l'état solide: en atmosphère $\mathrm{H}_{2}-\mathrm{CO}_{2}$, elle conduit en majorité à la formation de la sidérite alors qu'en atmosphère $\mathrm{N}_{2}-\mathrm{H}_{2}$, c'est la magnétite $\mathrm{Fe}_{3} \mathrm{O}_{4}$ qui est formée en quantité ( $\mathrm{ROH}$ et al., 2003 et 2006).

\subsubsection{Influence du manganèse, du phosphate, des sulfures et des nitrates sur le fer(II)}

Le fer et le manganèse subissent les mêmes transformations redox dans l'environnement aquatique (DAG HONGVE, 1997). Ces deux éléments ont le même comportement chimique : tout comme le fer(II), le manganèse(II) est instable dans les zones oxiques où il précipite sous forme de dioxyde de manganèse $\mathrm{MnO}_{2}$. Dans les zones anoxiques, ce dernier oxyde malheureusement le fer(II) selon la réaction :

$$
\begin{aligned}
& 2 \mathrm{Fe}^{2+}+\mathrm{MnO}_{2}+4 \mathrm{H}_{2} \mathrm{O} \\
& \quad \rightarrow 2 \mathrm{Fe}(\mathrm{OH})_{3}+\mathrm{Mn}^{2+}+2 \mathrm{H}^{+}
\end{aligned}
$$

En présence du phosphate et en l'absence du manganèse(II), il y a précipitation de la vivianite $\mathrm{Fe}_{3}\left(\mathrm{PO}_{4}\right)_{2}, 8 \mathrm{H}_{2} \mathrm{O}$ (DAG HONGVE, 1997; FREDERICHS et al., 2003). C'est par ce processus préjudiciable au fer(II) que le phosphate des eaux non manganifères est régulé.

Les lacs qui contiennent du fer et des sulfures en quantité ont généralement leurs sédiments pourvus de pyrite FeS. En présence de l'oxygène, le sulfure s'oxyde en sulfate. C'est donc dans la zone anoxique des lacs qu'il y a formation de pyrite, soit par combinaison directe de $\mathrm{HS}^{-}$avec $\mathrm{Fe}^{2+}$, soit indirectement à partir de $\mathrm{Fe}(\mathrm{OH})_{3}$ selon le mécanisme ci-après décrit par KONOVALOV et al. (2004) :

$$
\begin{aligned}
& 8 \mathrm{Fe}(\mathrm{OH})_{3}+\mathrm{HS}^{-}+15 \mathrm{H}^{+} \\
& \rightarrow 8 \mathrm{Fe}^{2+}+\mathrm{SO}_{4}^{2-}+20 \mathrm{H}_{2} \mathrm{O} \\
& \mathrm{Fe}^{2+}+2 \mathrm{HS}^{-} \rightarrow \mathrm{FeS}_{2}+\mathrm{H}_{2}
\end{aligned}
$$

L'oxydation anaérobie de $\mathrm{Fe}^{2+}$ limite également la quantité $\mathrm{du}$ fer(II) en solution des lacs. Elle se fait selon deux voies : en présence du $\mathrm{CO}_{2}$ et de la lumière selon la réaction suivante (EHRENREICH et WIDDEL, 1994)

$$
\begin{gathered}
4 \mathrm{Fe}^{2+}+\mathrm{CO}_{2}+11 \mathrm{H}_{2} \mathrm{O} \text { (lumière) } \\
\rightarrow 4 \mathrm{Fe}(\mathrm{OH})_{3}+\mathrm{CH}_{2} \mathrm{O}+8 \mathrm{H}^{+}
\end{gathered}
$$

et en présence des nitrates selon la réaction (SCHELOBOLINA et al., 2003; STRAUB et al.,1996)

$$
\begin{aligned}
& 10 \mathrm{Fe}^{2+}+2 \mathrm{NO}_{3}^{-}+24 \mathrm{H}_{2} \mathrm{O} \\
& \rightarrow 10 \mathrm{Fe}(\mathrm{OH})_{3}+\mathrm{N}_{2}+18 \mathrm{H}^{+}
\end{aligned}
$$


Ou encore

$$
2 \mathrm{Fe}^{2+}+\mathrm{NO}_{3}^{-}+2 \mathrm{H}^{+} \rightarrow 2 \mathrm{Fe}^{3+}+\mathrm{H}_{2} \mathrm{O}+\mathrm{NO}_{2}^{-}
$$

Bioxyde de manganèse, phosphate, sulfure et nitrates limitent de ce fait la quantité de fer(II) en solution des lacs.

\subsubsection{Origine de l'ion $\mathrm{HCO}_{3}^{-}$, précurseur de lion $\mathrm{CO}_{3}^{2-}$ / élément constitutif de $\mathrm{HCO}_{3}^{-}$}

\subsubsection{Source géochimique de l'ion $\mathrm{HCO}_{3}$}

$\mathrm{HCO}_{3}^{-}$peut provenir du gaz magmatique $\mathrm{CO}_{2}$ qui au contact de l'eau se dissous en $\mathrm{H}_{2} \mathrm{CO}_{3}$. Selon le $\mathrm{pH}$ de la solution, l'acide carbonique peut se dissocier en hydrogénocarbonate en libérant des protons selon les réactions suivantes :

$$
\begin{gathered}
\mathrm{CO}_{2}+\mathrm{H}_{2} \mathrm{O} \leftrightarrow \mathrm{H}_{2} \mathrm{CO}_{3} \\
\mathrm{H}_{2} \mathrm{CO}_{3} \leftrightarrow \mathrm{H}^{+}+\mathrm{HCO}_{3}^{-}
\end{gathered}
$$

L'hydrogénocarbonate en solution du lac ne provient pas que de la dissociation de $\mathrm{H}_{2} \mathrm{CO}_{3}$, mais peut être un produit d'altération des roches (NIA et al., 2009) :

$$
\begin{gathered}
\mathrm{CaCO}_{3}+\mathrm{CO}_{2}+\mathrm{H}_{2} \mathrm{O} \\
\leftrightarrow \mathrm{Ca}^{2+}+2 \mathrm{HCO}_{3}^{-}(\text {altération de la calcite }) \\
\mathrm{Mg}_{2} \mathrm{SiO}_{4}+4 \mathrm{CO}_{2}+2 \mathrm{H}_{2} \mathrm{O} \\
\leftrightarrow 2 \mathrm{Mg}^{2+}+4 \mathrm{HCO}_{3}^{-} \\
+\mathrm{SiO}_{2}(\text { altération de l'olivine }) \\
\leftrightarrow \mathrm{KAlSi}_{3} \mathrm{O}_{8}+2 \mathrm{CO}_{2}+2 \mathrm{H}_{2} \mathrm{O}^{2} \\
\leftrightarrow \mathrm{Al}_{2} \mathrm{Si}_{4} \mathrm{O}_{10}\left(\mathrm{OH}_{2}+2 \mathrm{~K}^{+}\right. \\
+2 \mathrm{HCO}_{3}^{-}+2 \mathrm{SiO}_{2}(\text { altération de l'orthose }) \\
\leftrightarrow \mathrm{CaAl}_{2} \mathrm{Si}_{2} \mathrm{O}_{8}+2 \mathrm{CO}_{2}+2 \mathrm{H}_{2} \mathrm{O} \\
\leftrightarrow \mathrm{Al}_{2} \mathrm{Si}_{2} \mathrm{O}_{5}\left(\mathrm{OH}_{4}+2 \mathrm{Ca}^{2+}\right. \\
+2 \mathrm{HCO}_{3}^{-}(\text {altération de l'anorthite }) \\
+\mathrm{Al}_{2} \mathrm{Si}_{2} \mathrm{O}_{5}\left(\mathrm{OH}_{4}+2 \mathrm{Na}^{+}\right. \\
+2 \mathrm{HCO}_{3}^{-}+4 \mathrm{SiO}_{2}(\text { altération de l'albite })
\end{gathered}
$$

4.1.2.2 Source biologique de l'ion $\mathrm{HCO}_{3}^{-}$

La fermentation bactérienne est un phénomène courant des milieux marins, océaniques et lacustres. Dans la zone oxique, la matière organique est dégradée avec dégagement de $\mathrm{CO}_{2}$, gaz dominant. Une fois dissous en $\mathrm{H}_{2} \mathrm{CO}_{3}$ il se transforme en $\mathrm{HCO}_{3}^{-}$en selon le $\mathrm{pH}$ de la solution du lac. Dans la zone anoxique, le méthane est le gaz dominant de la fermentation. L'acétogenèse est l'étape prédominante de la méthanogenèse et peut se résumer par la réaction générale cidessous (WHITICAR, 1999)

$$
\mathrm{CH}_{3} \mathrm{CO}_{2} \mathrm{H} \rightarrow \mathrm{CH}_{4}+\mathrm{CO}_{2}
$$

Une partie du méthane est ensuite oxydée en $\mathrm{CO}_{2}$ dans la zone oxique, une autre partie dans la zone anoxique par des bactéries spécifiques (GREINERT et al., 2001; LEIN, 2004; SAPOTA et al., 2006).

La production biogénique de $\mathrm{HCO}_{3}^{-}$en milieu anoxique s'explique mieux si on le considère comme étant un produit d'oxydation anaérobie de la matière organique constituée de carbone d'azote et de phosphore (MÜLLER et al., 2006) :

$$
\begin{aligned}
\mathrm{C}_{106} & \mathrm{H}_{263} \mathrm{O}_{110} \mathrm{~N}_{16} \mathrm{P}+84,8 \mathrm{NO}_{3}^{-} \\
\leftrightarrow & 106 \mathrm{HCO}_{3}^{-}+16 \mathrm{NH}_{4}^{+}+\mathrm{HPO}_{4}^{2-} \\
+ & 7,2 \mathrm{H}^{+}+42,4 \mathrm{~N}_{2}+42,4 \mathrm{H}_{2} \mathrm{O} \\
\mathrm{C}_{106} & \mathrm{H}_{263} \mathrm{O}_{110} \mathrm{~N}_{16} \mathrm{P}+424 \mathrm{FeOOH} \\
+ & 756 \mathrm{H}^{+} \leftrightarrow 106 \mathrm{HCO}_{3}^{-}+16 \mathrm{NH}_{4}^{+} \\
+ & \mathrm{HPO}_{4}^{2-}+424 \mathrm{Fe}^{2+}+636 \mathrm{H}_{2} \mathrm{O} \\
\mathrm{C}_{106} & \mathrm{H}_{263} \mathrm{O}_{110} \mathrm{~N}_{16} \mathrm{P}+53 \mathrm{SO}_{4}^{2-} \\
\leftrightarrow & 106 \mathrm{HCO}_{3}^{-}+16 \mathrm{NH}_{4}^{+}+\mathrm{HPO}_{4}^{2-} \\
+ & 53 \mathrm{HS}^{-}+39 \mathrm{H}^{+}
\end{aligned}
$$

En milieu aérobie, le phénomène est tout autre : plus de protons générés que d'hydrogénocarbonates formés (STUMM et MORGAN, 1996) :

$$
\begin{aligned}
& \mathrm{C}_{106} \mathrm{H}_{263} \mathrm{O}_{110} \mathrm{~N}_{16} \mathrm{P}+138 \mathrm{O}_{2} \leftrightarrow 106 \mathrm{HCO}_{3}^{-} \\
& +\mathrm{HPO}_{4}^{2-}+124 \mathrm{H}^{+}+16 \mathrm{NO}_{3}^{-}+16 \mathrm{H}_{2} \mathrm{O}
\end{aligned}
$$

L'ion $\mathrm{H}^{+}$étant l'unique cation compensateur des anions $\mathrm{HCO}_{3}^{-}, \mathrm{HPO}_{4}^{2-}$ et $\mathrm{NO}_{3}^{-}$, le bilan de cette dégradation bactérienne en milieu aérobie est :

$$
\begin{gathered}
\mathrm{C}_{106} \mathrm{H}_{263} \mathrm{O}_{110} \mathrm{~N}_{16} \mathrm{P}+138 \mathrm{O}_{2} \leftrightarrow 106 \mathrm{H}_{2} \mathrm{CO}_{3} \\
+\mathrm{H}_{3} \mathrm{PO}_{4}+16 \mathrm{HNO}_{3}+16 \mathrm{H}_{2} \mathrm{O}
\end{gathered}
$$


À partir de ce bilan on constate que la décomposition de la matière organique en présence de l'oxygène renforce l'acidité de la solution du lac, ce qui a pour effet de réduire considérablement la dissociation de $\mathrm{H}_{2} \mathrm{CO}_{3}$ en $\mathrm{HCO}_{3}^{-}$.

Ainsi, en milieu aérobie, c'est selon un mécanisme biologique (B) et chimique (C) qu'on obtient l'hydrogénocarbonate (mécanisme BC) alors qu'en milieu anaérobie, c'est par un mécanisme purement biologique (B) que se forme cet ampholyte:

Milieu aérobie:

$$
\begin{gathered}
\mathrm{MO}+\mathrm{O}_{2} \stackrel{\mathrm{B}}{\rightarrow} \mathrm{H}_{2} \mathrm{CO}_{3} \\
\mathrm{H}_{2} \mathrm{CO}_{3} \stackrel{\mathrm{C}}{\rightarrow} \mathrm{H}^{+}+\mathrm{HCO}_{3}^{-}
\end{gathered}
$$

Milieu anaérobie:

$$
\mathrm{MO}+\text { Oxydant } \stackrel{\text { B }}{\rightarrow} \mathrm{HCO}_{3}^{-}
$$

$\mathrm{HCO}_{3}^{-}$, formé en milieu anoxique, peut être considéré comme étant de l'hydrogénocarnate biologique strict, parce que produit uniquement par des micro-organismes, sans réaction chimique ultérieure. Par contre en milieu oxique, c'est $\mathrm{du} \mathrm{HCO}_{3}^{-}$biochimique.

\subsubsection{Conditions et mécanisme de formation de la sidérite}

De ce qui précède, on peut suggérer les différentes formes suivantes de sidérite selon l'origine de leurs éléments constitutifs: sidérite géochimique, sidérite biogénique et sidérite biogéochimique.

La sidérite biogénique doit sa formation à :

- la matière organique et au $\mathrm{CO}_{2}$ total qui doivent être disponibles -à l'absence des sulfures et

- à l'activité microbienne (BAHRIG, 1989; CANFIELD et al., 1992; CURTIS et al., 1986; KHIM et al.,1999; PEARSON, 1979; POSTMA, 1982; PYE, 1984; ZODROW et al., 1996).

Cette activité bactérienne en milieu anoxique induit un potentiel redox négatif favorable à la formation de la sidérite. C'est dans les eaux interstitielles que le phénomène a lieu, décrit aussi bien dans les milieux marins peu profonds que dans les plaines inondables, les delta et les lacs (COLEMAN et RAISWELL, 1993; CURTUS et al., 1986; DUAN et al., 1996; FREDERICHS et al., 2003; KRYLOV et al., 2008; MOORE et al.,1992; MORTIMER et COLEMAN, 1997; MOZLEY et CAROTHERS, 1992; ROMANEK et al., 2003; ZHANG et al.,1998 et 2001). Dans cette étude, nous intéressons en particulier à la sidérite endogénique qui précipite dans la colonne d'eau des lacs.

HSU et KELTS (1978) étudiant la sédimentation chimique de la mer Noire (BlacK Sea) suggérait pour la première fois que la sidérite relevée dans les sédiments provenait probablement de la colonne d'eau (sidérite endogénique). Selon ces auteurs, la disponibilité du fer réactif et de la matière organique en l'absence des sulfures dans un environnement anoxique auraient amélioré les conditions de saturation, favorisant la précipitation de la sidérite. Des études menées sur deux lacs volcaniques camerounais (Nyos et Monoun), après émission de gaz mortels, ont révélé la précipitation de la sidérite dans la colonne d'eau (BERNARD et SYMONDS, 1989; SIGURDSSON et al., 1987). Il apparait que le dégazage spontané, l'évaporation due au réchauffement (GLENN, 2008; RAJAN et al., 1996) et le dégazage forcé d'un lac sont des facteurs qui peuvent favoriser la saturation en solides carbonatés. Le potentiel redox négatif de la solution du lac, sans être une condition suffisante, serait nécessaire à la précipitation de la sidérite. Dans le cas de la sidérite biogénique, ce potentiel négatif est induit par l'activité bactérienne (COLEMAN et RAISWELL, 1993; KRYLOV et al., 2008; POSTMA, 1982). Lorsque ces conditions sont réunies, la sidérite précipite selon la réaction suivante :

$$
\mathrm{Fe}^{2+}+2 \mathrm{HCO}_{3}^{-} \leftrightarrow \mathrm{FeCO}_{3}+\mathrm{H}_{2} \mathrm{CO}_{3}
$$

Prenant en compte ces considérations et se référant à la mer Noire, RAJAN et al. (1996) ont développé un modèle thermodynamique de précipitation endogène de la sidérite. Plus encore, certains auteurs ont expérimenté la précipitation de $\mathrm{FeCO}_{3}$ soit à partir d'un précurseur métastable à température et pression élevées (BRUNO et al., 1992; CAROTHERS et al.,1988; WERSIN et al.,1989) soit par recristallisation des précurseurs à des températures comprises entre $35^{\circ} \mathrm{C}$ et $70^{\circ} \mathrm{C}$ (JOHNSON, 1990). SINGER et STUMM (1970) ont réussi à précipiter la sidérite inorganique entre $17^{\circ} \mathrm{C}$ et $30^{\circ} \mathrm{C}$ à la pression atmosphérique à partir d'un protocole expérimental brièvement décrit, sans information sur l'évolution du milieu. Tout récemment, JIMENEZ-LOPEZ et ROMANEK (2004) ont étudié la cinétique de précipitation de la sidérite à $25^{\circ} \mathrm{C}$ et 1 atmosphère à pression de $\mathrm{CO}_{2}$ relativement faible et en atmosphère d'azote (milieu anaérobie). Le modèle expérimental qu’ils utilisent est basé sur la technique " chemostat ", technique qui nécessite l'ensemencement du milieu avec quelques cristaux de produit à précipiter (MORSE, 1974). Cependant, la précipitation de la sidérite est sensible aux conditions réductrices et sa cinétique est lente. Pour cette raison, ces auteurs ont développé une technique alternative basée sur la nucléation homogène, génératrice de semences propices à la précipitation de la sidérite. 
4.1.4 Expérimentation de la précipitation du carbonate de fer (sidérite) inspirée du diagramme potentiel- $p H$

Notre dispositif expérimental plus simplifié tient compte essentiellement des conditions réductrices et anaérobies du milieu, par analogie à la sidérite biogénique qui ne s'obtient que dans ces conditions. La figure 1 indique le domaine de stabilité thermodynamique du carbonate de fer : c'est à des potentiels négatifs (milieu réducteur) que précipite ce solide carbonaté. Le recours à un réducteur chimique (dithionite de sodium) permet d'atteindre ces potentiels. Le milieu réactionnel a été réalisé tel que décrit dans la partie 1.2.1. La filtration s'est déroulée en atmosphère d'azote, dans une boîte à gants pour éviter l'évolution de la forme solide carbonatée vers la forme hydroxyde ou oxyde. Les résultats d'analyse chimique avant et

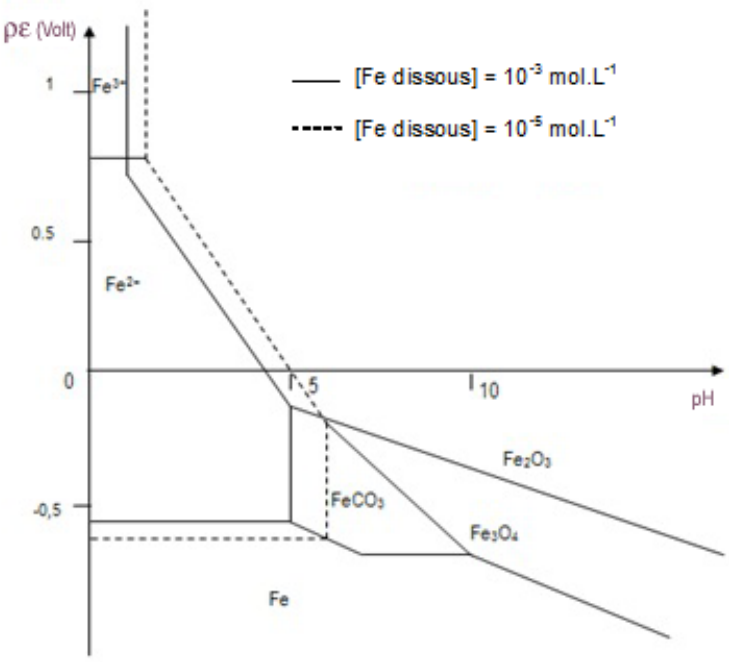

Figure 1. Diagramme potentiel-pH du système $\left[\mathrm{Fe}, \mathrm{CO}_{2}, \mathrm{H}_{2} \mathrm{O}\right]$ Potential-pH diagram of the system $\left[\mathrm{Fe}, \mathrm{CO}_{2}, \mathrm{H}_{2} \mathrm{O}\right]$

Tableau 1. Analyse chimique des solutions avant introduction de $\mathrm{NaOH}, \mathrm{Na}_{2} \mathrm{~S}_{2} \mathrm{O}_{4} 0,5 \mathrm{mM}$, et après précipitation du carbonate de fer (sidérite) ; les concentrations sont en mmol. $\mathrm{L}^{-1}$ et les potentiels redox $\rho \varepsilon$ en $\mathrm{mV} /$ ECS (électrode au calomel saturée) ; $\mathrm{T}=25^{\circ} \mathrm{C}$.

Table 1. Chemical analysis of solutions before introduction of $\mathrm{NaOH}, \mathrm{Na}_{2} \mathrm{~S}_{2} \mathrm{O}_{4} 0.5 \mathrm{mM}$, and after precipitation of iron carbonate (siderite); concentrations are expressed in mmol. $L^{-1}$ and redox potential, $\rho \varepsilon$, in $m V /$ $S C E$ (saturated calomel electrode); $T=25^{\circ} \mathrm{C}$

\begin{tabular}{|c|c|c|c|c|c|c|c|c|c|c|c|c|c|c|}
\hline & $\begin{array}{l}\rho \mathcal{E} \\
\text { (i) }\end{array}$ & $\begin{array}{l}\rho \mathcal{E} \\
\text { (f) }\end{array}$ & $\begin{array}{l}\mathrm{pH} \\
\text { (i) }\end{array}$ & $\begin{array}{c}\mathrm{pH} \\
\text { (f) }\end{array}$ & $\begin{array}{l}\mathrm{CO}_{2 \mathrm{~T}} \\
\text { (i) }\end{array}$ & $\begin{array}{c}\mathrm{CO}_{2 \mathrm{~T}} \\
(\mathrm{f})\end{array}$ & $\begin{array}{l}\mathrm{Fe}^{2+} \\
\text { (i) }\end{array}$ & $\begin{array}{l}\mathrm{Fe}^{2+} \\
(\mathrm{f})\end{array}$ & $\begin{array}{l}\mathrm{Ca}^{2+} \\
\text { (i) }\end{array}$ & $\begin{array}{c}\mathrm{Ca}^{2+} \\
(\mathrm{f})\end{array}$ & $\begin{array}{l}\mathrm{Mg}^{2+} \\
\text { (i) }\end{array}$ & $\begin{array}{l}\mathrm{Mg}^{2+} \\
\text { (f) }\end{array}$ & $\begin{array}{c}\mathrm{Ca} / \mathrm{Fe} \\
\text { (i) }\end{array}$ & $\begin{array}{l}\mathrm{Mg} / \mathrm{Fe} \\
\text { (i) }\end{array}$ \\
\hline E1 & $\begin{array}{c}+ \\
100\end{array}$ & -590 & 6,2 & 7,4 & 23 & 3,80 & 21,20 & 2,1 & 0 & 0 & 0 & 0 & 0 & 0 \\
\hline E2 & $\begin{array}{c}+ \\
110\end{array}$ & $\begin{array}{c}- \\
610\end{array}$ & 6,7 & 7,2 & 12,50 & 11,20 & 1,88 & 1,20 & 0 & 0 & 1,7 & 1,2 & 0 & 0,92 \\
\hline E3 & $\begin{array}{c}+ \\
88\end{array}$ & - & 6,6 & 7,1 & 12,50 & 3,48 & 9,00 & 0,10 & 0,45 & 0,36 & 2,5 & 2,1 & 0,05 & 0,83 \\
\hline E4 & $\begin{array}{c}+ \\
100\end{array}$ & $\begin{array}{c}- \\
620\end{array}$ & 6,5 & 7,7 & 12,40 & 11,50 & 1,86 & 1,15 & 3,93 & 3,78 & 0 & 0 & 2,11 & 0 \\
\hline E5 & $\begin{array}{c}+ \\
120\end{array}$ & - & 6,7 & 7,0 & 12,50 & 10,40 & 2 & 0,2 & 0,3 & 0,1 & 2,1 & 2 & 0,15 & 1,05 \\
\hline E6 & $\begin{array}{c}+ \\
130\end{array}$ & $\begin{array}{c}- \\
605\end{array}$ & 6,8 & 7,2 & 12,40 & 10,20 & 2,5 & 0,3 & 0,15 & 0,04 & 3,50 & 3,20 & 0,06 & 1,4 \\
\hline E7 & $\begin{array}{c}+ \\
104\end{array}$ & $\begin{array}{c}- \\
595\end{array}$ & 6,7 & 6,8 & 12,50 & 10,00 & 3,5 & 1,05 & 0,30 & 0,12 & 0,71 & 0,65 & 0,08 & 0,20 \\
\hline E8 & $\begin{array}{c}+ \\
95\end{array}$ & $\begin{array}{c}- \\
612\end{array}$ & 6,5 & 7,5 & 12,50 & 10,38 & 1,85 & 0,04 & 1,97 & 1,66 & 1,95 & 1,4 & 1,06 & 1,07 \\
\hline E9 & $\begin{array}{c}+ \\
117\end{array}$ & $\begin{array}{c}- \\
589\end{array}$ & 6,8 & 7,1 & 12,50 & 11,13 & 2,00 & 0,80 & 1,70 & 1,48 & 0 & 0 & 0,85 & 0 \\
\hline $\begin{array}{l}\mathrm{E} 10 \\
50^{\circ} \mathrm{C}\end{array}$ & $\begin{array}{l}+ \\
60\end{array}$ & $\begin{array}{c}- \\
420\end{array}$ & 6,4 & 6,8 & 14,50 & 13,50 & 2,00 & 1,02 & 3,98 & 3,93 & 0 & 0 & 1,99 & 0 \\
\hline
\end{tabular}


après précipitation sont confinés dans le tableau 1; le tableau 2 regroupe les données d'analyse des précipités et le tableau 3 les paramètres calculés de la maille élémentaire. La figure 2 donne la morphologie des cristaux observée au microscope électronique à balayage (SEM).
Analyse par diffraction des rayons X (DRX) : le décalage des distances réticulaires, $\mathrm{d}$, et l'absence de pics de fractionnement des principales réflexions $(102,104,110,113,202,116)$ observés dans les diffractogrammes montrent clairement que c'est la solution solide de $\mathrm{FeCO} 3-\mathrm{CaCO} 3$ qui précipite et non

Tableau 2. Analyse des précipités du fer en solution $\left(\mathrm{T}=25^{\circ} \mathrm{C}\right.$; ss : solution solide $\left.\mathrm{Fe}_{1}-\mathrm{x} \mathrm{Ca}_{\mathrm{x}} \mathrm{CO}_{3}\right)$ Table 2. Analysis of iron precipitates in solution $\left(\mathrm{T}=25^{\circ} \mathrm{C}\right.$; ss: solid solution $\left.\mathrm{Fe}_{1}-x \mathrm{Ca}_{x} \mathrm{CO}_{3}\right)$

\begin{tabular}{lcccccccc}
\hline Essais & $\begin{array}{c}\text { Qté }(\mathrm{mg}) \\
\text { précipité })\end{array}$ & $\begin{array}{c}\mathrm{Fe} \\
\mathrm{mmol} / \mathrm{L}\end{array}$ & $\begin{array}{c}\mathrm{Ca} \\
\mathrm{mmol} / \mathrm{L}\end{array}$ & $\begin{array}{c}\mathrm{Mg} \\
\mathrm{mmol} / \mathrm{L}\end{array}$ & $\% \mathrm{Fe}^{\mathrm{II}}$ & $\% \mathrm{Ca}$ & $\% \mathrm{Mg}$ & DRX \\
\hline E1 & 2215,6 & 19,6 & - & 0.0 & 100 & 0,0 & 0.0 & Sidérite \\
E2 & 78,9 & 0,68 & - & 0.0 & 100 & 0,0 & 0.0 & Sidérite \\
E3 & 910,6 & 7,92 & 0,08 & 0.0 & 99,0 & 1,0 & 0.0 & Sidérite $^{\text {Es }}$ \\
E4 & 82,4 & 0,67 & 0,11 & 0.0 & 81,7 & 18,3 & 0.0 & Sidérite $^{\text {ss }}$ \\
E5 & 197,2 & 1,70 & 0,18 & 0.0 & 89,5 & 10,5 & 0.0 & Sidérite $^{\text {ss }}$ \\
E6 & 243,6 & 2,11 & 0,10 & 0.0 & 95,0 & 5,0 & 0.0 & Sidérite $^{\text {ss }}$ \\
E7 & 278,4 & 2,42 & 0,16 & 0.0 & 93,0 & 7,1 & 0.0 & Sidérite $^{\text {ss }}$ \\
E8 & 197,2 & 1,75 & 0,31 & 0.0 & 85,0 & 15,0 & 0.0 & Sidérite $^{\text {ss }}$ \\
E9 & 127,6 & 1,10 & 0,20 & 0.0 & 83,9 & 16,1 & 0.0 & Sidérite $^{\text {ss }}$ \\
E10,50 ${ }^{\circ} \mathrm{C}$ & 104,4 & 0,91 & 0,04 & 0.0 & 94,7 & 5,3 & 0.0 & Sidérite $^{\text {ss }}$ \\
\hline
\end{tabular}
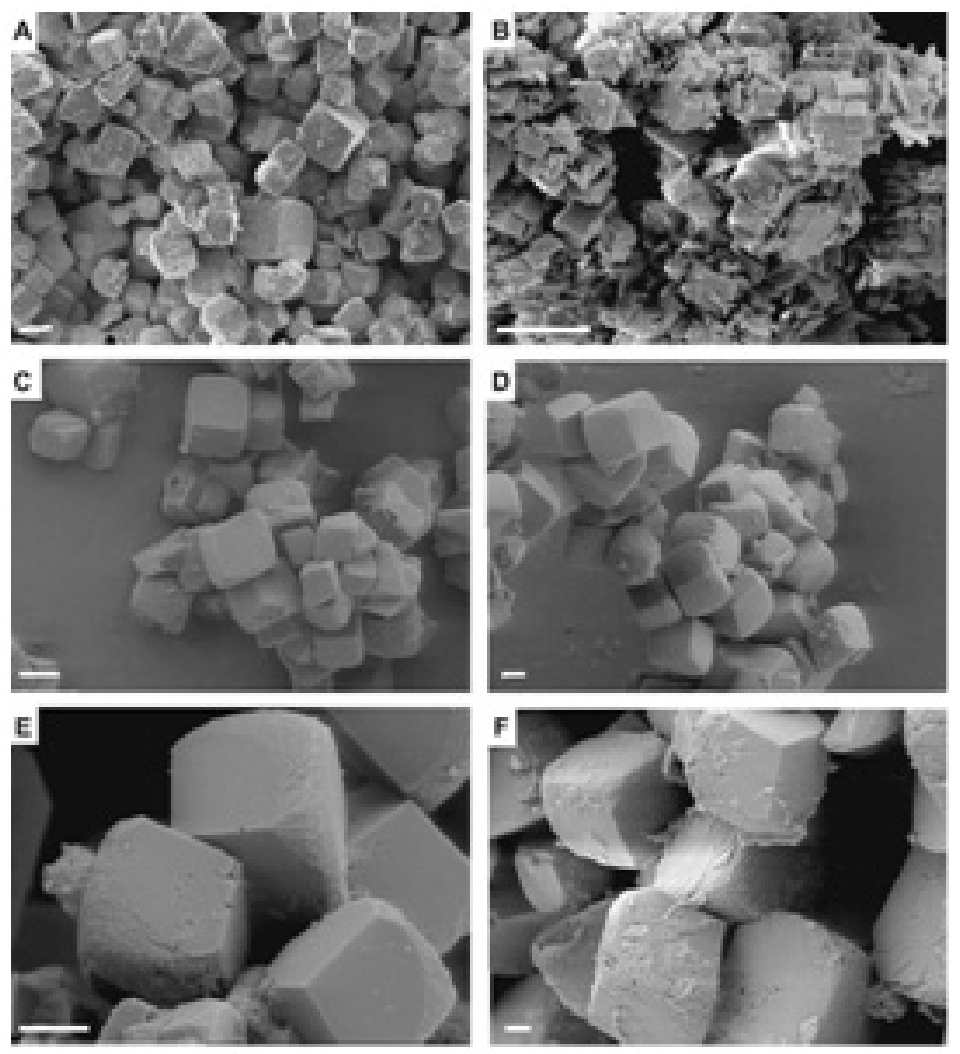

Figure 2. Microphotographies SEM des précipités du fer : (A) E1; (B) E2; (C) E3; (D) E6; (E) E7; (F) E9. Barres d'échelle $2 \mu \mathrm{m}$ SEM photomicrographs for iron precipitates in: (A) E1; (B) E2; (C) E3; (D) E6; (E) E7; (F) E9. Scale bars $2 \mu \mathrm{m}$ 
Tableau 3. Paramètres de la maille élémentaire des précipités du fer

Table 3. Unit cell parameter for iron precipitates

\begin{tabular}{lcc}
\hline Essais & $\mathrm{a}\left(\mathrm{A}^{\circ}\right)$ & $\mathrm{C}\left(\AA^{\circ}\right)$ \\
\hline E1 & 4,7213 & 15,4622 \\
E2 & 4,7237 & 15,4708 \\
E3 & 4,7186 & 15,3654 \\
E4 & 4,7505 & 15,535 \\
E5 & 4,7289 & 15,6984 \\
E6 & 4,6893 & 15,5703 \\
E7 & 4,7195 & 15,3764 \\
E8 & 4,7403 & 15,5685 \\
E9 & 4,7397 & 15,6118 \\
E10 & 4,7025 & 15,5143 \\
\hline
\end{tabular}

des phases séparées (e.g. sidérite et calcite), particulièrement illustrées par les expériences E6 et E10 (tableau 2). Certaines expériences (E6, E7, E10) ont conduit à la sidérite contenant 5 à $7 \%$ mol $\mathrm{CaCO}_{3}$ avec des longueurs d'axe «a ", comprises entre 4,6893 et 4,7186 $\mathrm{A}^{\circ}$ (Tableau 3). D'autres, par contre, ont conduit à la sidérite contenant 15 à $18 \% \mathrm{~mol} \mathrm{CaCO}_{3}$ (E4, E8, E9) avec des axes, a, significativement importants en longueur $\left(4,7397\right.$ à 4,7505 $\left.\mathrm{A}^{\circ}\right)$. En effet, l'incorporation considérable du calcium crée des tensions au sein du réseau cristallin de la sidérite de base (E1, E2) et explique la déviation significative des réflexions de plus forte intensité. L'absence du magnésium dans les précipités (Tableau 2) montre une incorporation préférentielle du calcium dans le réseau de la sidérite même en présence d'une concentration importante de magnésium (Tableau 1, E6). Ces données confirment celles de ROMANEK et al. (2009) qui obtiennent des résultats similaires dans des conditions expérimentales pourtant différentes.

Analyse SEM : le microscope électronique à balayage montre que le solide précipité est un rhomboèdre, les faces 104 caractéristiques du cristal (sidérite, solution solide $\mathrm{Fe}_{1-\mathrm{x}} \mathrm{CaCO}_{3}$ ) étant bien définies ainsi que les arrêtes et les sommets (Figure 2). La longueur des arrêtes de la maille rhomboédrique de la sidérite pure (E1 et E2) ou pratiquement pure (E3) est comprise entre 2 et $3 \mu \mathrm{m}$ (Figure 3, A-B-C); pour des solutions solides contenant 5 à $7 \% \mathrm{~mol} \mathrm{CaCO}_{3}$ (E5, E7, E10) elle varie de 3 à $4 \mu \mathrm{m}$ (Figure 2, D ), et de 6 à $14 \mu \mathrm{m}$ (Figure 2, F) pour des solutions solides contenant 15 à $18 \% \mathrm{~mol} \mathrm{CaCO}_{3}$ (E4, E8, E9).

Les analyses RDX et SEM montrent ainsi qu'en milieu réducteur et bicarbonaté calcique, le fer(II) précipite sous forme de solution solide carbonatée dans laquelle le solvant de base est le fer, ce qui est en accord avec la littérature (FREDERICHS et al., 2003; KRYLOV et al., 2008; RAJAN et al.,1996). Plusieurs expériences menées dans des conditions identiques aux précédentes, mais sans ajout de réducteur chimique ont révélé une diminution considérable du fer(II) sans modification quelconque du $\mathrm{CO}_{2}$ total : les précipités seraient des hydroxydes ou des oxydes de fer. Le potentiel redox est de ce fait un paramètre important de la précipitation du carbonate de fer à l'état pur ou sous forme de solution solide.

\subsection{Mécanisme de précipitation endogène de la rhodochrosite lacustre $\mathrm{MnCO}_{3}$}

L'origine de l'ion carbonate, élément constitutif de $\mathrm{MnCO}_{3}$ a été évoquée au paragraphe 1.2. L'origine de $\mathrm{Mn}^{2+}$ est à chercher dans le cycle même du manganèse. Le mécanisme de réduction bactérienne de $\mathrm{MnO}_{2}$ en $\mathrm{Mn}^{2+}$, lequel ne fait pas l'unanimité, est discuté dans cette partie de l'étude. Il en de même de la stabilité du manganèse(II).

\subsubsection{Cycle du manganèse des lacs}

Tout comme le fer, le manganèse est impliqué dans le cycle redox des environnements marins oxiques/anoxiques : sulfure, oxygène, nitrate et ammonium forment avec ces deux métaux un système redox complexe qui régit la zone de transition suboxique - anoxique (LEWIS et LANDING, 1991; MURRAY et al., 1995; OGUZ et al., 2001). Le manganèse dissous $\mathrm{Mn}(\mathrm{II})$ est stable dans la zone anoxique des lacs où la concentration de l'oxygène ne dépasse pas $1 \mathrm{mg} / \mathrm{L}$ (CANFIELD et al., 1992; DAG HONGVE, 1997); oxydé en $\mathrm{MnO}_{2}$ dans la zone oxique, il est rétabli dans la zone anoxique par réduction bactérienne et le cycle recommence (BALISTRIERI et al.,1992; KAWASHIMA et al.,1988).

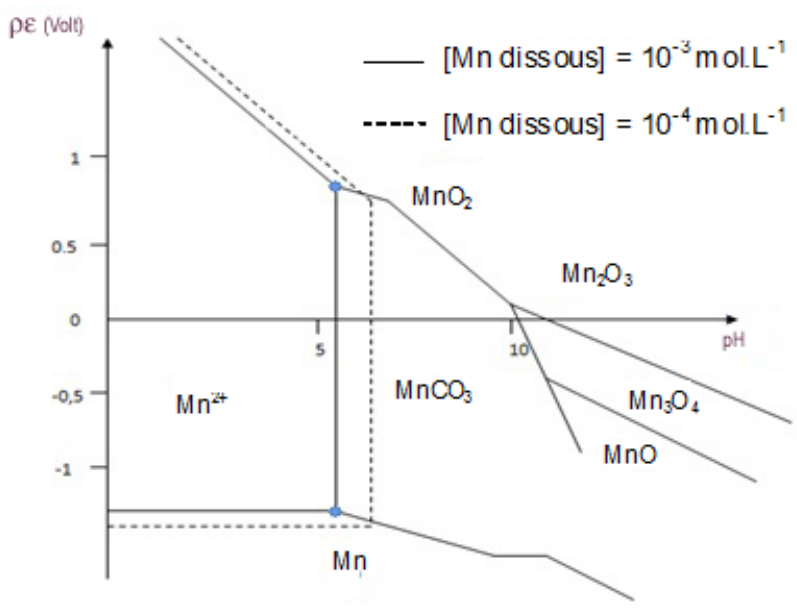

Figure 3. Diagramme potentiel-pH du système $\left[\mathrm{Mn}, \mathrm{CO}_{2}, \mathrm{H}_{2} \mathrm{O}\right]$ Potential-pH diagram of the system [Mn, $\left.\mathrm{CO}_{2}, \mathrm{H}_{2} \mathrm{O}\right]$ 
4.2.1.1 Influence des sulfures et des phosphates sur la stabilité de $\mathrm{Mn}^{2+}$

4.2.1.1.1 Action des sulfures

En présence des sulfures, $\mathrm{Mn}^{2+}$ précipite en $\mathrm{MnS}$ selon le mécanisme ci-dessous proposé par KONOLOV et al., (2004). En milieu suffisamment pourvu d'espèces carboniques, le dioxyde de manganèse est réduit en carbonate de manganèse (équation 30).

$$
\begin{gathered}
\mathrm{MnO}_{2}+\mathrm{HS}^{-}+3 \mathrm{H}^{+} \rightarrow \mathrm{Mn}^{2+}+\mathrm{S}^{0}+2 \mathrm{H}_{2} \mathrm{O} \\
\mathrm{Mn}^{2+}+2 \mathrm{HS}^{-} \rightarrow \mathrm{MnS}_{2}+\mathrm{H}_{2} \\
\mathrm{MnO}_{2}+\mathrm{HS}^{-}+\mathrm{HCO}_{3}^{-}+2 \mathrm{H}^{+} \\
\rightarrow \mathrm{MnCO}_{3}+\mathrm{S}^{0}+2 \mathrm{H}_{2} \mathrm{O}
\end{gathered}
$$

L'équation (30) s'obtient également par combinaison de l'équation (28) avec l'équation (31) suivante :

$$
\mathrm{Mn}^{2+}+2 \mathrm{HCO}_{3}^{-} \leftrightarrow \mathrm{MnCO}_{3}+\mathrm{H}_{2} \mathrm{CO}_{3}
$$

\subsection{Action des phosphates}

En présence des phosphates, le manganèse(II) précipite sous forme de $\mathrm{MnHPO}_{4}$ selon la réaction

$$
\mathrm{Mn}^{2+}+\mathrm{HPO}_{4}^{2-} \rightarrow \mathrm{MnHPO}_{4}
$$

DAG HONGVE (1997), étudiant le cycle du fer, du manganèse et du phosphate dans un lac stagnant nettement plus riche en $\mathrm{Mn}(\mathrm{II})$ qu'en $\mathrm{Fe}(\mathrm{II})$, constate l'absence de la vivianite $\mathrm{Fe}_{3}\left(\mathrm{PO}_{4}\right)_{2} \cdot 8 \mathrm{H}_{2} 0$ dans les sédiments, mais la présence de $\mathrm{FeOOH}$ et $\mathrm{MnHPO}_{4}$. C'est dire combien le cycle du manganèse peut influencer celui du fer au point où la vivianite ne soit plus l'élément régulateur des phosphates dans les zones anoxiques.

\subsubsection{Influence de la nitrification- dénitrification sur le cycle du manganèse}

$\mathrm{MnO}_{2}$ précipité dans la zone oxique traverse la frontière oxique-anoxique et sombre dans la zone anoxique où l'ammoniaque $\mathrm{NH}_{4}^{+}$est stable. L'oxydation anaérobie de $\mathrm{NH}_{4}^{+}$en $\mathrm{NO}_{3}^{-}$par des bactéries nitrifiantes s'accompagne de la réduction de $\mathrm{MnO}_{2}$ en $\mathrm{Mn}^{2+}$ selon la réaction (33)

$$
\mathrm{NH}_{4}^{+}+4 \mathrm{MnO}_{2}+6 \mathrm{H}^{+} \rightarrow 4 \mathrm{Mn}^{2+}+\mathrm{NO}_{3}^{-}+5 \mathrm{H}_{2} \mathrm{O}
$$

La réduction du nitrate en azote est également possible (dénitrification) :

$$
2 \mathrm{NO}_{3}^{-}+5 \mathrm{Mn}^{2+}+4 \mathrm{H}_{2} \mathrm{O} \rightarrow 5 \mathrm{MnO}_{2}+\mathrm{N}_{2}+8 \mathrm{H}^{+}
$$

Par le premier mécanisme (33) on comprend pourquoi $\mathrm{Mn}^{2+}$ est plus concentré dans l'hypolimnion que dans l'épilimnion des lacs stratifiés à forte teneur en $\mathrm{NH}_{4}^{+}$.

Le second mécanisme (34) montre à quel point la dénitrification bactérienne est préjudiciable à $\mathrm{Mn}^{2+}$.

\subsubsection{Origine biogénique de $\mathrm{Mn}^{2+}$ : Réduction biologique de $\mathrm{MnO}_{2}$}

Bon nombre d'études ont été consacrées à l'oxydation bactérienne de $\mathrm{Mn}(\mathrm{II})$ (TEBO, 1991). Par contre, le mécanisme de la réduction de ses oxydes, en particulier le dioxyde de manganèse $\mathrm{MnO}_{2}$ est peu documenté et assez controversé. Pour certains, c'est en présence de l'oxygène que la réduction a lieu (CALVERT et PEDERSON, 1996). Pour d'autres, ce n'est possible qu'en milieu anoxique réducteur (DELIAN et al., 1992; FORCE et MAYNARD, 1991; FRAKES et BOLTON, 1992; JENKYNS et al., 1991; OKITA et SHANKS, 1992). Selon NEUMANN et al. (2002), $\mathrm{Mn}^{2+}$ s'oxyde en $\mathrm{MnO}_{2}$ dans les zones oxiques. Une fois parvenu dans les zones anoxiques, l'oxyde est réduit en $\mathrm{Mn}^{2+}$ par des bactéries réductrices du manganèse oxyde. $\mathrm{Mn}^{2+}$ diffuse ensuite dans les eaux interstitielles, zones de forte alcalinité due à la forte minéralisation de la matière organique. La rhodochrosite entachée de calcium (Ca-rhodochrosite) se formerait juste au-dessus de la surface des sédiments où l'eau interstitielle est sursaturée en ce carbonate solide (KULIK et al., 2000). Deux remarques peuvent être dégagées des travaux de ces auteurs : la dégradation bactérienne de la matière organique (pourvoyeur de $\mathrm{CO}_{2}$, précurseur de $\mathrm{HCO}_{3}^{-}$) est une étape séparée de la réduction de $\mathrm{MnO}_{2}$; les réactions de formation de la rhodochrosite avec transfert d'électrons et de protons à $\mathrm{MnO}_{2}$ ne sont pas décrites.

Nous proposons ci-dessous un mécanisme similaire à celui que nous avons suggéré plus avant pour la réduction bactérienne de $\mathrm{Fe}(\mathrm{OH})_{3}$ (paragraphe 2.1.1.2.2) :

$$
\begin{gathered}
\mathrm{MO} \rightarrow \mathrm{e}+\mathrm{H}^{+}+\text {métabolites } \\
\mathrm{ADP}+\mathrm{Pi} \rightarrow \text { ATP } \\
\mathrm{MnO}_{2}+2 \mathrm{e}+4 \mathrm{H}^{+} \text {manganèse réductase } \rightarrow \\
\mathrm{Mn}^{2+}+2 \mathrm{H}_{2} \mathrm{O}
\end{gathered}
$$

\subsubsection{Autres sources de $\mathrm{Mn}(\mathrm{II})$ :}

4.2.1.4.1 Réduction de $\mathrm{MnO}_{2}$ par Fe ${ }^{2+}$

$\mathrm{MnO}_{2}$ est un oxydant énergique et peut oxyder toute substance dont le pouvoir oxydant est inférieur au sien. Abondant dans ce sens, KONOLOV et al. (2004) ont proposé 
un mécanisme probable de réduction de $\mathrm{MnO}_{2}$ par le fer(II) dans les zones suboxiques et anoxiques de la mer Noire :

$$
\begin{aligned}
& \mathrm{MnO}_{2}+2 \mathrm{e}+4 \mathrm{H}^{+} \text {manganèse réductase } \\
& \rightarrow \mathrm{Mn}^{2+}+2 \mathrm{H}_{2} \mathrm{O}
\end{aligned}
$$

Il s'agit ici d'une réduction bactérienne de $\mathrm{MnO}_{2}$ qui s'accompagne d'une oxydation de fer(II) avec libération de protons, lesquels stabilisent $\mathrm{Mn}^{2+}$ formés (milieu acide anoxique)

\subsection{Décomposition acide de $\mathrm{Mn} 3 \mathrm{O} 4$}

Il n'est pas rare de rencontrer dans les couches profondes de certains lacs du manganèse(II) et de l'oxygène dissous en quantité considérable. GREEN et al. (2004), constatant un accroissement local de $\mathrm{Mn}^{2+}$ dans les eaux profondes oxygénées du lac Vanda, proposent le mécanisme suivant :

$$
\begin{aligned}
& \mathrm{Mn}_{3} \mathrm{O}_{4}+6 \mathrm{H}^{+} \\
& \rightarrow 3 \mathrm{Mn}^{2+}+\frac{1}{2} \mathrm{O}_{2}+3 \mathrm{H}_{2} \mathrm{O}
\end{aligned}
$$

\subsubsection{Conditions et mécanisme de formation et de précipitation de la rhodochrosite}

Les origines des éléments constitutifs, telles que décrites plus avant, apportent d'avantage de précisions à l'endogénèse du carbonate de manganèse $\mathrm{MnCO}_{3}$ : la rhodochrosite endo-géochimique et la rhodochrosite endo-biogéochimique précipitent tous dans la colonne d'eau, mais par un mécanisme différent de par l'origine de leurs éléments constitutifs. La rhodochrosite biogénique stricte serait celle dont les deux éléments constitutifs seraient tous d'origine biologique et qui se formerait dans les eaux interstitielles.

MIZANDRONTSEV (1975) était le premier à penser que les carbonates des sédiments du lac Baïkal pouvaient être la rhodochrosite eu égard au taux élevé de manganèse qui s'y trouvait. Contrairement à la sidérite, la rhodochrosite authigénique se forme dans tous les environnements anoxiques soufrés et non soufrés (GLASBY et SCHULTZ, 1999). On la retrouve aussi bien dans les sédiments marins (CHOW et al., 2000; MORAD et AL-AaSM, 1997; NEUMANN et al., 2002) que dans les sédiments lacustres (KRYLOV et al., 2008; STEVENS et al., 2000). Sa formation survient lorsque le milieu est riche en matière organique et contient du manganèse à l'état libre ou sous forme d'oxydes (FREDERICHS et al., 2003; SHAOFENG et al., 2010). Toutefois la médiation biologique joue un rôle important aussi bien dans l'authigènèse de la rhodochrosite (NEALSON et SAFFARINI, 1994) que dans l'authigènèse de la sidérite ( KONHAUSER, 1998).

\subsubsection{Expérimentation de la précipitation du carbonate de manganèse (rhodochrosite) inspirée du diagramme potentiel-pH}

Nous nous intéressons en particulier au carbonate de manganèse qui précipite en solution du lac (DAG HONGVE, 1997) bien que la forme oxy-hydroxyde soit la plus décrite (DEAN et DONER, 2011; DEAN et al., 2003). Le diagramme potentiel-pH du système $\left(\mathrm{Mn}^{2+}, \mathrm{CO}_{2}, \mathrm{H}_{2} \mathrm{O}\right)$, figure 3 , permet de localiser le domaine de stabilité thermodynamique du carbonate de manganèse. Contrairement au carbonate de fer, le potentiel redox n'est pas un paramètre déterminant : c'est aussi bien en milieu oxydant qu'en milieu réducteur que précipite le carbonate de manganèse. Par contre c'est en milieu anoxique suffisamment pourvu de $\mathrm{HCO}_{3}^{-}$et saturé en $\mathrm{MnCO}_{3}$ que précipiterait le manganèse selon l'équation (31). Le tableau 4 récapitule les données d'analyses chimiques de deux eaux synthétiques fabriquées selon la méthode décrite plus avant (Méthodologie) et désaérées par barbotage d'azote, auxquelles est ajouté du chlorure de manganèse. Après élévation du $\mathrm{pH}$ à la soude jusqu'à obtention d'une solution blanchâtre, l'équilibre est sûrement atteint au bout de $24 \mathrm{~h}$ d'agitation : la quantité de $\mathrm{Mn}^{2+}$ et de $\mathrm{Ca}^{2+}$ retranchés de la solution équivaut pratiquement à la diminution du $\mathrm{CO}_{2}$ total dans les deux expériences (E1 et E2). La réduction du calcium, bien qu'infime (E1), fut inévitable après plusieurs opérations. Au regard des analyses chimiques, le précipité serait soit une solution solide $\mathrm{Mn}_{1 \text {. }}$ ${ }_{x} \mathrm{Ca}_{\mathrm{x}} \mathrm{CO}_{3}$ riche en manganèse (E1), soit un mélange de deux carbonates solides $\mathrm{MnCO}_{3}$ et $\mathrm{CaCO}_{3}$ (E2). La diffraction des rayons $\mathrm{x}$ des précipités recueillis révèle tantôt une solution solide carbonatée enrichie à 93 \% environ en manganèse (E1),

Tableau 4. Analyse chimique des solutions de manganèse avant introduction de $\mathrm{NaOH}$ et après précipitation du carbonate

\begin{tabular}{|c|c|c|c|c|c|c|c|c|c|c|}
\hline & $\begin{array}{l}\mathrm{pH} \\
\text { (i) }\end{array}$ & $\begin{array}{l}\mathrm{pH} \\
\text { (f) }\end{array}$ & $\begin{array}{l}\mathrm{CO}_{2 \mathrm{~T}} \\
\text { (i) }\end{array}$ & $\begin{array}{l}\mathrm{CO}_{2 \mathrm{~T}} \\
\text { (f) }\end{array}$ & $\begin{array}{l}\mathrm{Mn}^{2+} \\
\text { (i) }\end{array}$ & $\begin{array}{l}\mathrm{Mn}^{2+} \\
(\mathrm{f})\end{array}$ & $\begin{array}{c}\mathrm{Ca}^{2+} \\
\text { (i) }\end{array}$ & $\begin{array}{l}\mathrm{Ca}^{2+} \\
(\mathrm{f})\end{array}$ & $\begin{array}{l}\mathrm{Ca} / \mathrm{Mn} \\
\text { (i) }\end{array}$ & DRX \\
\hline E1 & 6,94 & 7,46 & 6,12 & 5,96 & 0,93 & 0,79 & 2,36 & 2,33 & 2,98 & $\begin{array}{l}\text { Solution solide } \\
\mathrm{Mn}_{1-\mathrm{x}} \mathrm{Cax}_{3} \mathrm{CO}_{3}\end{array}$ \\
\hline E2 & 6,75 & 8,14 & 10,58 & 6,00 & 1,01 & 0,01 & 3,95 & 0,42 & 3,91 & $\begin{array}{c}\text { Calcite + solution solide } \\
\qquad \mathrm{Mn}_{1-\mathrm{x}} \mathrm{Ca}_{\mathrm{x}} \mathrm{CO}_{3}\end{array}$ \\
\hline
\end{tabular}
de manganèse; les concentrations sont en mmol $\cdot \mathrm{L}-1$; $\mathrm{T}=25^{\circ} \mathrm{C}$.

Table 4. Chemical analysis of manganese solutions before introduction of $\mathrm{NaOH}$ and after precipitation of manganese carbonate; concentrations are expressed in mmolL-1; T $=25^{\circ} \mathrm{C}$. 
tantôt un mélange calcite-solution solide $\mathrm{Mn}_{1-\mathrm{x}} \mathrm{Ca}_{\mathrm{x}} \mathrm{CO}_{3}$ (E2). Ces données sont en accord avec les prévisions de la littérature (LIM et al., 2004; NEUMANN et al., 2002).

\subsection{Mécanisme de précipitation endogène de la calcite et de l'aragonite lacustres}

\subsubsection{Origine de l'ion $\mathrm{Ca}^{2+}$, élément constitutif de $\mathrm{CaCO}_{3}$}

Le calcium est le cinquième élément chimique le plus abondant de la croûte terrestre ; il est introduit dans le système d'eau douce sous l'action de la météorisation des roches calcaires (DAY,1963) ; La combustion de combustibles fossiles et les effluents industriels sont deux sources non négligeables de calcium des lacs : c'est le cas des Grands Lacs (INTERNATIONAL REFERENCE GROUP, 1977; UPPER LAKES REFERENCE GROUP, 1976)

\subsubsection{Précipitation de la calcite et de l'aragonite en solution des lacs}

La nucléation, étape importante du mécanisme de précipitation du carbonate de calcium, est bien documentée (LEBRON et SUAREZ, 1996; MORSE et al., 2007; MURPHY et al.,1983; ROSSMANN, 1980; STABEL, 1996). L'influence de cette précipitation sur le cycle du carbone des lacs eutrophisés est remarquable. Pour cette raison, plusieurs études ont mis l'accent sur la formation, sur la réaction, les processus de sédimentation et les dépôts de $\mathrm{CaCO}_{3}$ dans les lacs (EFFLER, 1984; EFFLER et JOHNSON, 2007; ELFIL et ROQUES, 2001; GAL et al., 2002; HOUSE, 1984; KELTS et HSÜ, 1978; STABEL, 1986). La température et le rapport $\mathrm{Mg}: \mathrm{Ca}$ déterminent la composition et la variété cristallographique de carbonate de calcium précipité : calcite, aragonite, solutions solides $\mathrm{Ca}_{1-\mathrm{x}} \mathrm{Mg}_{\mathrm{x}} \mathrm{CO}_{3}$ ont été ainsi décrites (DEAN, 2009; MORSE et al., 2007; MÜLLER ,1972; SHAPLEY et al., 2002). La calcite est la variété cristalline de carbonate de calcium la plus stable à température et pression ambiantes, l'aragonite étant stable à haute pression et la vatérite étant thermodynamiquement instable (CÖLFEN, 2003; JOHANESS et PUHAN, 1971; NAN et al., 2007).Cependant, ZHAODONG et al. (2008), dans une étude récente ont obtenu la vatérite à partir de l'aragonite en présence d'un surfactant. Étudiant les lacs alcalins de la vallée de Ovando dans l'ouest du Montana, SHAPLEY et al.(2002) ont montré une forte correspondance entre les anomalies du climat (évaporation des lacs) et l'endogénèse de l'aragonite précipité. DEAN (2009), réalisant une étude sur les lacs Utah et Idaho note trois grands intervalles de carbonates précipités en solution des lacs et déposés dans les sédiments pendant l'holocène. D'après cet auteur, l'intervalle carbonaté majoritaire (aragonite) coïncide avec les climats continentaux chauds interglaciaux qui se sont succédés.

Dans cette étude, nous nous intéressons à la précipitation endogène de $\mathrm{CaCO}_{3}$, sans action biologique, guidée uniquement par la saturation du lac en ce carbonate et dont les éventuelles causes ont été rappelées à l'introduction (DEAN, 2009; DEAN et DONER, 2011; DEAN et SCHWALB, 2002; SHAPLEY et al., 2002). La contribution à cette saturation des espèces solubles non chargées $\left(\mathrm{CaCO}_{3}{ }^{0}\right.$ en l'occurrence) n'est pas à négliger (BOLLINGER et al.,1988; GAL et al., 1996). Quand le produit des activités des ions $\mathrm{Ca}^{2+}$ et $\mathrm{CO}_{3}^{2-}$ devient supérieur à la constante de dissociation de $\mathrm{CaCO}_{3}$ qui est de 10-8,48 (PLUMMER et al., 1978), il précipite du carbonate de calcium selon la réaction ci-dessous (ELFIL et ROQUES, 2001; LANGMUIR, 1997) :

$$
\mathrm{Ca}^{2+}+2 \mathrm{HCO}_{3}^{-} \rightarrow \mathrm{CaCO}_{3}+\mathrm{H}_{2} \mathrm{CO}_{3}
$$

L'un des éléments constitutifs de ce carbonate solide peut être d'origine géochimique (calcium par exemple) et l'autre d'origine biologique (dégradation anaérobie de la matière organique en $\mathrm{HCO}_{3}^{-}$). Ce serait alors de la calcite ou de l'aragonite biogéochimique si l'on tient compte de l'origine des éléments qui les constituent. Les constituants peuvent être d'origine géochimique avec une nucléation biologique de $\mathrm{CaCO}_{3}$ : ce serait encore de la calcite biogéochimique, et c'est bien le cas du lac Constance, la nucléation étant assurée par des algues (STABEL,1996). À notre sens, la calcite biogénique stricte serait celle dont la nucléation calcique est biologique, les éléments constitutifs étant d'origine biologique. La précipitation du carbonate de calcium dans les solutions modèles et dans les eaux naturelles dépend essentiellement du degré de saturation du milieu en ce carbonate (PROFT et STUTTER, 1993). Par conséquent, il importe peu que le milieu soit oxydant ou réducteur. Des essais menés à $25^{\circ} \mathrm{C}$ à partir d'échantillons d'eau synthétisée selon la méthode décrite plus avant et sans précaution aucune (milieu aéré ou désaéré) ont conduit à la précipitation de $\mathrm{CaCO}_{3}$ d'après les analyses chimiques. Ces expériences aux résultats prévisibles (non présentés ici) n'ont rien d'attrayant en elles-mêmes, cependant permettent tout au moins de comprendre que seuls les lacs durs alcalins ( $\mathrm{pH}$ supérieur à 8 ) précipitent le carbonate de calcium dans la colonne d'eau lorsque les conditions qui favorisent leur saturation en ce carbonate sont réunies (DEAN et SCHWALB, 2002; MÜLLER et al., 2006).

\subsection{Mécanisme de précipitation endogène des carbonates de magnésium}

Le carbonate de magnésium est soit anhydre (magnésite $\left.\mathrm{MgCO}_{3}\right)$, soit hydraté $\left(\mathrm{MgCO}_{3} \cdot \mathrm{H}_{2} \mathrm{O}\right.$, $\left.\mathrm{MgCO}_{3} \cdot 2 \mathrm{H}_{2} \mathrm{O}, \quad \mathrm{MgCO}_{3} \cdot 3 \mathrm{H}_{2} \mathrm{O}, \quad \mathrm{MgCO}_{3} \cdot 5 \mathrm{H}_{2} \mathrm{O}\right) . \quad \mathrm{La}$ littérature fait état de ces diverses formes cristallines réparties dans certains sédiments lacustres, le plus souvent combinées au calcium (DEAN, 1999; QUERALT et al., 1997). LAST et De DECKKER (1990) décrivent la plupart de solutions de lacs australiens comme étant fortement sursaturées 
thermodynamiquement en calcite, aragonite, dolomite $\mathrm{CaMg}\left(\mathrm{CO}_{3}\right)_{2}$, magnésite et hydromagnésite. Le mécanisme de précipitation endogène des carbonates de magnésium serait similaire à celui du carbonate de calcium : Ce sont des lacs alcalins à dureté magnésienne dominante $\left(\mathrm{Mg}^{2+}\right.$ nettement plus élevée que $\mathrm{Ca}^{2+}$ ) qui, une fois saturée en carbonates de magnésium, précipite en solution des lacs selon la réaction (39) :

$$
\mathrm{Mg}^{2+}+2 \mathrm{HCO}_{3}^{-} \leftrightarrow \mathrm{MgCO}_{3}+\mathrm{H}_{2} \mathrm{CO}_{3}
$$

La magnésite $\mathrm{MgCO}_{3}\left(\mathrm{~K}_{\mathrm{s}}=10^{-8,04} \mathrm{JOHNSON}\right.$ et al., 1992) de même ordre de solubilité que la calcite $\mathrm{CaCO}_{3}\left(\mathrm{Ks}=10^{-}\right.$ ${ }^{8,48}$ PLUMMER et al.,1978) ne peut précipiter seule en présence du calcium. Par ailleurs le rayon atomique peu différent des atomes $\mathrm{Ca}^{2+}$ et $\mathrm{Mg}^{2+}$, la structure cristalline identique de leur carbonate (rhomboédrique), leurs charges et leurs électronégativités similaires sont autant de facteurs favorables à la formation de solution solide $\mathrm{Ca}_{1-\mathrm{x}} \mathrm{Mg}_{\mathrm{x}} \mathrm{CO}_{3}$ tant décrites dans la littérature (DEAN, 2009; LAST et De DECKKER, 1990; MORSE et al., 2007; MÜLLER et al.,1972; QUERALT et al.,1997).

\subsection{Phénomènes stabilisants et phénomènes destructifs des carbonates solides des lacs}

Dans ce qui précède, les mécanismes de précipitation endogène de solides carbonatés majeurs des lacs ont été revus et complétés. Pour en faire des indicateurs climatiques, leur stabilité doit être de longue durée après sédimentation, ce qui n'est pas toujours le cas. Cette partie de l'étude s'intéresse aux phénomènes néfastes et favorables à la stabilisation des carbonates solides précipités dans la colonne d'eau des lacs.

\subsubsection{Oxydation aérobie de la matière organique : action des protons $H^{+}$}

La dissolution de la calcite dans la colonne d'eau ou dans les sédiments dépend de la production de protons que génère la décomposition aérobie de la matière organique (MÜLLER et al., 2006). L'équilibre (22) illustre bien le phénomène. Ces protons agissent soit à l'état (40), soit combinés au bicarbonate sous forme d'acide carbonique (41) :

$$
\begin{gathered}
\mathrm{MCO}_{3}+2 \mathrm{H}^{+} \rightarrow \mathrm{M}^{2+}+\mathrm{CO}_{2}+\mathrm{H}_{2} \mathrm{O} \\
\mathrm{MCO}_{3}+\mathrm{H}_{2} \mathrm{CO}_{3} \leftrightarrow \mathrm{M}^{2+}+2 \mathrm{HCO}_{3}^{-} \\
\mathrm{M}: \mathrm{Ca}, \mathrm{Mg}, \text { Fe ou Mn }
\end{gathered}
$$

Alors que la production de la matière organique (eutrophisation par exemple) élève le $\mathrm{pH}$ de l'eau et provoque la précipitation du carbonate de calcium, la décomposition de la matière organique augmente l'acidité de l'hypolimnion propice à la dissolution de ce carbonate (DEAN, 1999). Les carbonates de fer, de manganèse et de magnésium subissent les mêmes contraintes.

\subsubsection{Oxydation biologique du carbonate de fer $\mathrm{FeCO}_{3}$}

Certaines bactéries acidophiles sont capables d'oxyder le fer(II) à l'état solide (KAPPLER et NEWMAN, 2004; PISAPIA et al., 2007). ZHANG et al. (2009), dans une étude expérimentale récente, rapportent la minéralisation biologique du citrate ferrique et de la nontronite (phyllosilicate riche en fer) par des micro-organismes réducteurs $\mathrm{de} \mathrm{Fe}^{3+}$, également celle de la vivianite, sid érite et pyrite par des micro-organismes oxydatifs de $\mathrm{Fe}^{2+}$. Par cette étude et pour la première fois, ils ont montré qu'en milieu anoxique, il existe un cycle du fer dans lequel le fer(II) et le fer (III) sont à l'état solide. Cependant, les mécanismes de réduction et d'oxydation biologiques de ces solides ferreux et ferriques n'ont pas été décrits par ces auteurs. À notre sens, le mécanisme de réduction du citrate ferrique et de la nontronite serait le même que celui proposé dans cette étude pour la réduction d'hydroxyde de fer et d'oxyde de manganèse. L'oxydation de la vivianite, de $\mathrm{FeS}$ et de $\mathrm{FeCO}_{3}$ obéirait à un mécanisme beaucoup plus complexe, d'après ZHANG et al. (2009). La réflexion suivante que nous menons sur $\mathrm{FeCO}_{3}$ est applicable aux autres carbonates évoqués dans notre étude.

\subsubsection{Oxydation en présence des nitrates}

L'oxydation biologique de $\mathrm{FeCO}_{3}$, donneur d'électrons, nécessite un accepteur qui peut être du nitrate $\mathrm{NO}_{3}^{-}$comme c'est le cas pour l'oxydation anoxique de $\mathrm{Fe}^{2+}$ (SCHELOBOLINA et al., 2003; STRAUB et al.,1996). Cependant il n'est pas encore prouvé que les bactéries oxydatives de $\mathrm{Fe}^{2+}$ dépendantes des nitrates soient capables de conserver l'énergie issue de l'oxydation de Fe(II) à l'état solide (KAPPLER et STRAUB, 2005).Toutefois, des bactéries anaérobies thermophiles telles que Thermoanaerobacter ethanolicus isolées majoritairement par ZHANG et al. (2009) dans leur milieu de culture sont capables d'oxyder le fer(II) et de réduire le fer (III) à l'état solide. Nous proposons le mécanisme suivant en milieu anoxique :

4.5.2.1.1 $\mathrm{FeCO}_{3}$ est oxydé en $\mathrm{Fe}^{3+}$ et $\mathrm{NO}_{3}^{-}$est réduit en $\mathrm{N}_{2}$

$$
\begin{gathered}
\mathrm{FeCO}_{3}+2 \mathrm{H}^{+} \text {ferrioxydase } \\
\rightarrow \mathrm{Fe}^{3+}+\mathrm{e}^{-}+\mathrm{CO}_{2}+\mathrm{H}_{2} \mathrm{O} \\
2 \mathrm{NO}_{3}^{-}+12 \mathrm{H}^{+}+10 \mathrm{e}^{-} \rightarrow \mathrm{N}_{2}+6 \mathrm{H}_{2} \mathrm{O}
\end{gathered}
$$

Ici, la bactérie est perçue comme une biomasse fixée sur un support solide $\left(\mathrm{FeCO}_{3}\right)$ en milieu anoxique et en présence des nitrates. Les deux réactions simultanées nécessitent un contact entre la bactérie et le carbonate de fer sans lequel le transfert 
des électrons du donneur $\mathrm{FeCO}_{3}$ à l'accepteur $\mathrm{NO}_{3}^{-}$n'est pas possible. Une fois le contact établi, l'oxydation de $\mathrm{FeCO}_{3}$ est activée par l'enzyme ferrioxydase qui assure également le transfert de l'électron sur le nitrate. Ce transfert pourrait être assuré par une enzyme autre : l'enzyme transférase. L'énergie d'activation de la réaction serait considérable avec la sidérite cristallisée (faible contenu énergétique). Le contact optimal s'obtiendrait donc avec la bemmelenite qui est une sidérite amorphe colloïdale, bien que chargée négativement. Il est à remarquer que le système redox consomme les protons du milieu, ce qui n'est pas le cas pour la réduction biologique de $\mathrm{Fe}(\mathrm{OH})_{3}$, où le système génèrent ses protons . C'est donc en milieu anoxique et bien acide que cette oxydation se ferait si c'est le cas. Il est à remarquer également que la réduction d'une mole de nitrate s'accompagnerait de l'oxydation de 5 moles de $\mathrm{FeCO}_{3}$.

4.5.2.1.2 $\mathrm{FeCO}_{3}$ est oxydé en $\mathrm{Fe}^{3+}$ et $\mathrm{NO}_{3}^{-}$est réduit en $\mathrm{NO}_{2}^{-}$

Dans ce cas, la réaction (42) est couplée à la réaction (44) suivante :

$$
\mathrm{NO}_{3}^{-}+2 \mathrm{H}^{+}+2 \mathrm{e}^{-} \rightarrow \mathrm{NO}_{2}^{-}+\mathrm{H}_{2} \mathrm{O}
$$

Cette fois la réduction d'une mole de $\mathrm{NO}_{3}^{-}$engendre l'oxydation de deux moles de $\mathrm{FeCO}_{3}$ toujours avec consommation des protons du milieu et non du système redox. S’il en était ainsi, le milieu devrait être acide, mais moins que dans le premier cas.

4.5.2.1.3 $\mathrm{FeCO}_{3}$ est oxydé en $\mathrm{Fe}(\mathrm{OH})_{3}$ et $\mathrm{NO}_{3}^{-}$est réduit en $\mathrm{NO}_{2}^{-}$

A la réaction (44), nous couplons la réaction (45) suivante:

$$
\begin{aligned}
& \mathrm{FeCO}_{3}+2 \mathrm{H}_{2} \mathrm{O} \text { ferrioxydase } \\
& \quad \rightarrow \mathrm{e}^{-}+\mathrm{H}^{+}+\mathrm{Fe}(\mathrm{OH})_{3}+\mathrm{CO}_{2}
\end{aligned}
$$

Les électrons et les protons échangés proviennent du système redox, l'oxydation d'une mole de $\mathrm{FeCO}_{3}$ entrainant la réduction d'une demi-mole de $\mathrm{NO}_{3}^{-}$. Le système redox s'autoentretient.

4.5.2.1.4 $\mathrm{FeCO}_{3}$ est oxydé en $\mathrm{Fe}(\mathrm{OH})_{3}$ et $\mathrm{NO}_{3}^{-}$est réduit en $\mathrm{N}_{2}$.

La réaction d'oxydation-réduction qui a lieu dans ce cas s'obtient en couplant les réactions (43) et (45). La réduction d'une mole de nitrate s'accompagne de l'oxydation de 5 moles de $\mathrm{FeCO}_{3}$; à la différence du premier mécanisme (paragraphe 5.2.1.1), le système redox produit ses protons. Toutefois, le milieu devrait être acide eu égard au léger déficit protonique qu'accuse le système redox.

\subsubsection{Oxydation en présence des phosphates ou des sulfates}

Les bactéries sulfato-réductrices sont également capables de réduire $\mathrm{PO}_{4}^{3-}$ (autre accepteur d'électrons) en phosphine
$\mathrm{PH}_{3}$ (ROELS et VERSTRAETE, 2001). Il est donc possible que l'oxydation de $\mathrm{Fe}^{2+}$ soit couplée à la réduction de $\mathrm{PO}_{4}^{3-}$ , surtout que cette dernière est impliquée dans la corrosion du fer (ROELS et VERSTRAETE, 2001).Par ailleurs la réduction de $\mathrm{SO}_{4}^{2-}$ (autre accepteur d'électrons) en $\mathrm{SO}_{3}^{2-}$ par des bactéries oxydatives de $\mathrm{Fe}^{2+}$ est possible (ZHANG et al., 2009). Le milieu de culture bactérienne de ZHANG et al. (2009) contient toutes ses entités chimiques : présence de sulfates $\left(\mathrm{NH}_{4}\right)_{2} \mathrm{SO}_{4}$ et $\mathrm{MgSO}_{4}$, et de phosphates $\mathrm{K}_{2} \mathrm{HPO}_{4}$ et $\mathrm{KH}_{2} \mathrm{PO}_{4}$. Nous inspirant de ce milieu, nous proposons les mécanismes ci-après qui dépendent de la forme oxydée du carbonate de fer $\left(\mathrm{Fe}^{2+}\right.$ ou $\left.\mathrm{Fe}(\mathrm{OH})_{3}\right)$ :

4.5.2.2.1 Oxydation de $\mathrm{FeCO}_{3}$ en $\mathrm{Fe}^{3+}$ et réduction de $\mathrm{PO}_{4}^{3-}$ en $\mathrm{PH}_{3}$

Le mécanisme y afférent s'obtient en combinant la réaction (42) avec la réaction (46) suivante :

$$
\mathrm{PO}_{4}^{3-}+11 \mathrm{H}^{+}+8 \mathrm{e}^{-} \rightarrow \mathrm{PH}_{3}+4 \mathrm{H}_{2} \mathrm{O}
$$

Ce cas s'apparente à celui déjà évoqué (paragraphe 5.2.1.1) : forte consommation des protons du milieu, le système redox n'en produisant aucunement. Le milieu doit être acide pour entretenir le système redox.

4.5.2.2.2 Oxydation de $\mathrm{FeCO}_{3}$ en $\mathrm{Fe}(\mathrm{OH})_{3}$ et réduction de $\mathrm{PO}_{4}^{3-}$ en $\mathrm{PH}_{3}$

Le couplage des équations (45) et (46) rend compte du mécanisme. La réduction d'une mole de $\mathrm{PO}_{4}^{3-}$ entraine l'oxydation de 8 moles de $\mathrm{FeCO}_{3}$ en milieu acide.

4.5.2.2.3 Oxydation de $\mathrm{FeCO}_{3}$ en $\mathrm{Fe}^{3+}$ et réduction de $\mathrm{SO}_{4}^{2-}$ en $\mathrm{SO}_{3}^{2-}$

La réaction (42) et la réaction suivante (47) rendent compte du phénomène.

$$
\mathrm{SO}_{4}^{2-}+2 \mathrm{H}^{+}+2 \mathrm{e}^{-} \rightarrow \mathrm{SO}_{3}^{2-}+\mathrm{H}_{2} \mathrm{O}
$$

L'oxydation d'une mole de $\mathrm{FeCO}_{3}$ consomme autant de protons produits hors système redox que la réduction d'une mole de $\mathrm{SO}_{4}^{2-}$. Par conséquent, c'est en milieu acide qu'une oxydation-réduction biologique de cette nature pourrait avoir lieu.

4.5.2.2.4 Oxydation de $\mathrm{FeCO}_{3}$ en $\mathrm{Fe}(\mathrm{OH})_{3}$ et réduction de $\mathrm{SO}_{4}^{2-}$ en $\mathrm{SO}_{3}^{2-}$

Les réactions (45) et (47) traduisent les faits dans pareil cas : Les protons et les électrons échangés proviennent du système redox qui s'auto-entretient.

\subsubsection{Déplacement d'équilibre}

$$
\mathrm{CaCO}_{3}+\mathrm{H}_{2} \mathrm{CO}_{3} \leftrightarrow \mathrm{Ca}^{2+}+2 \mathrm{HCO}_{3}^{-}
$$

L'équilibre (13) est celui qui caractérise toutes les eaux naturelles, connu sous l'appellation équilibre calco-carbonique. Lorsqu'il se déplace vers la droite, on dit que l'eau est agressive 
(KAMGAING, 2003b; LEGRAND et POIRIER, 1976). S'il se déplace vers la gauche l'eau est dite incrustante. Une eau agressive attaque le carbonate de calcium, mais également tout matériau mis à son contact : l'intoxication au plomb d'origine hydrique, conséquence de la circulation d'eau agressive dans des tuyauteries en plomb est bien décrite (BEAUSOLEIL et BRODEUR, 2007; LEROY, 1994). Une eau agressive qui attaque le métal est susceptible d'attaquer le carbonate de ce métal. L'équilibre (13) étendu aux carbonates métalliques devient:

$$
\begin{gathered}
\mathrm{MCO}_{3}+\mathrm{H}_{2} \mathrm{CO}_{3} \leftrightarrow \mathrm{M}^{2+}+2 \mathrm{HCO}_{3}^{-} \\
\mathrm{M}: \mathrm{Ca}, \mathrm{Fe} \text { ou } \mathrm{Mn}
\end{gathered}
$$

En résumé, la stabilité ou la vulnérabilité des carbonates solides endogènes des lacs dépend du caractère de l'eau : une eau incrustante ou à l'équilibre stabiliserait d'avantage ces carbonates alors qu'une eau agressive aurait l'effet inverse (déplacement de l'équilibre (48) vers la droite).

\subsubsection{Influence de l'évaporation et du dégazage spontané ou forcé sur l'endogènèse et la stabilité des carbonates solides des lacs}

Toute action susceptible de modifier la concentration de la solution du lac en espèces dissoutes peut conduire à sa saturation et provoquer la précipitation entre autres d'oxydes ou de carbonates. L'action la plus régulière est l'évaporation du lac suite au réchauffement ; le dégazage spontané ou forcé suite à un retournement des eaux ou à un dégazage du lac respectivement est une action à considérer également. Lorsque ces phénomènes adviennent, l'eau change de composition et même de caractère (agressif, incrustant). En général, une élévation de température peut conduire à l'évaporation qui rend l'eau incrustante, c'est-àdire prompte à précipiter des carbonates solides (équilibre 48). L'évaporation artificielle d'un lac (dégazage forcé) n'est pas sans conséquences sur le caractère de l'eau. En effet, il existe une quantité d'acide carbonique qui maintient l'équilibre (13), connu sous l'appellation " acide carbonique équilibrant de l'eau » (LEGRAND et POIRIER, 1976). Pour éviter la rupture de cet équilibre, il convient d'éliminer uniquement la partie d'acide qui rendrait l'eau agressive, connue sous le nom d'acide carbonique agressif ou excédentaire. Lorsque le dégazage affecte le $\mathrm{CO}_{2}$ équilibrant, l'équilibre (13) se déplace vers la gauche où l'eau devient incrustante. Nos travaux antérieurs effectués avant dégazage ont montré que les eaux du lac Nyos avaient un caractère agressif alors que celles de Monoun étaient incrustantes (KAMGAING, 2003b). Ces deux lacs dévastateurs (KLING et al., 1987) constituent toujours un risque majeur pour l'homme et l'environnement et sont en phase de dégazage (HALBWACHS et al., 2004; KLING et al., 2005; KUSAKABE et al., 2008; SCHMID et al., 2006). Lorsque le $\mathrm{CO}_{2}$ équilibrant du lac Nyos sera éliminé, les eaux deviendront incrustantes avec pour conséquence une baisse d'alcalinité et de dureté doublée d'un envasement du lac par des carbonates solides (déplacement de l'équilibre 47 vers la gauche). Cette modification de la composition de l'eau pourrait provoquer une fois de plus le dégazage spontané tant redouté suite à une rupture d'équilibre des strates. Le même phénomène se produirait au lac Monoun où les eaux deviendraient plus incrustantes qu'avant. Ainsi, l'évaporation et le dégazage non contrôlé d'un lac auraient tendance à rendre ses eaux incrustantes favorables à la précipitation et à la rémanence des carbonates solides, mais néfastes à la stabilité du lac.

\section{CONCLUSION}

Plusieurs causes sont à l'origine de la précipitation des carbonates de fer, de manganèse ou de calcium en solution des lacs. L'évaporation due au réchauffement ou encore le dégazage spontané ou forcé favoriseraient la saturation du lac en ces carbonates. La revue de la littérature faite dans cette étude a permis de faire le point sur l'état des mécanismes de leur précipitation dans la colonne d'eau (carbonates endogéniques) et de leur formation par des micro-organismes dans les zones anoxiques et dans les eaux interstitielles (carbonates biogéniques). La prise en compte de l'origine de leurs éléments constitutifs (géochimique ou biologique) dans le processus de leur formation ou de leur précipitation apporterait plus de précision à l'endogènèse et à l'authigénèse des carbonates solides des milieux lacustres. Le caractère de l'eau (agressif ou incrustant) aurait une influence sur la précipitation et sur la stabilité des carbonates formés ou précipités.

\section{REMERCIEMENTS}

Nous remercions le Ministère de l'Enseignement Supérieur du Cameroun pour la prime spéciale accordée trimestriellement aux chercheurs du supérieur. Également, l'université de Rennes I pour la microanalyse des précipités.

\section{RÉFÉRENCES BIBLIOGRAPHIQUES}

BAHRIG, B. (1985). Paleo-environment information from deep water siderite (Lake of Laach, west Germany). Dans: Lacustrine Petroleum source rocks. FLEET, A.J., K. KELTS et M.R. TALBOT (Editeurs), Geol. Soc. Spec., 40, 153-158.

BAHRIG, B. (1989). Stable isotope composition of siderite as an indicator of the paleoenvironmental history of oil shale 
Lakes. Palaeogeogr. Palaeoclimatol. Palaeoecol., 70,139-151.

BALISTRIERI, L.S., J.W. MURRAY et B. PAUL (1992). The cycling of iron and manganese in the water column of Lake Sammamish, Washington. Limnol. Oceanogr., 37, 510-528.

BEAUSOLEIL, M. et J. BRODEUR (2007). Le plomb dans l'eau potable sur l'île de Montréal. État de situation et évaluation des risques à la santé. Agence de la santé et des services sociaux de Montréal, Québec, 59 p.

BERNARD, A.et R.B. SYMONDS (1989). The significance of siderite in the sediments from Lake Nyos, Cameroon. J. Volcan. Geotherm. Res., 39,187-194.

BOLLINGER, J.C., V. DELUCHAT, J.Y. GAL, N. GACHE and Y. FOVET (1988). Repartition of various soluble and insoluble species in supersaturated solutions. Pure \&Appl. Chem., 70, 1921-1924.

BRUNO, J., P. WERSIN et W. STUMM (1992). On the influence of carbonate in mineral dissolution: II. The solubility of $\mathrm{FeCO} 3(\mathrm{~s})$ at $25^{\circ} \mathrm{C}$ and 1 atm total pressure. Geochim. Cosmochim. Acta, 56, 1149-1155.

CALLENDER, E. et L. GRANINA (1992). Transition metal geochemistry of sedimentary pore fluids associated with hydrothermal activity in Lake Baikal. Dans: Water-Rock Interaction. KHARAKA Y.K. et A.S. MAEST (Editeurs), Brookfiel, Vt, pp. 621-626.

CALVERT, S.E. et T.F. PEDERSON (1996). Sedimentary geochemistry of manganese: Implications for the environment of the formation of manganiferous black shales. Econ. Geol., 91, 36-47.

CAROTHERS, W.W., L.H. ADAMI et R.J. ROSENBAUER (1988). Experimental oxygen isotope fractionation between siderite-water and phosphoric acid liberated CO2-siderite. Geochim. Cosmochim. Acta, 52, 2445-2450.

CANFIELD, D.E., R. RAISWELL et S. BOTTRELL (1992). The reactivity of sedimentary iron minerals toward sulfide. Am. J. Sci., 292, 659-683.

COLEMAN, M.L. et R. RAISWELL (1993). Microbial mineralization of organic matter: Mechanisms of selforganization and inferred rates of precipitation of diagenetic minerals. Phil. Trans. R. Soc. Lond. A., 344, 69-87.

CÖLFEN, H. (2003). Precipitation of carbonates: recent progress in controlled production of complex shapes: selfassembly. Current opinion in Colloid and Interface Science, 8, 23-31.
CURTIS, C.D.et M.L. COLEMAN (1986). Controls on the precipitation of early diagenetic calcite, dolomite and siderite concretions in complex depositional sequences. Dans: Roles of Organic Matter in Sediment Diagenesis. GAUTIER D.L. (Editeur), SEPM Special Publication, 38, pp. 23-33.

HONGVE, D. (1997). Cycling of iron, manganese and phosphate in a meromictic lake. Limnol. Oceanogr., 42, 635-647.

DAVISON, W. (1993). Iron and manganese in lakes. Earth Sci. Rev. 34, 119-163.

DAY, E.H. (1963). The chemical elements in nature. George C. Harrap \& Co, London, U.K., 372 p.

DEAN, W.E. (1999). The carbon cycle and Biogeochemical Dynamics in lake sediments. J. Paleolimnology, 21, 375393.

DEAN, W.E. (2009). Endogenic carbonate sediments in Bear Lake, Utah and Idaho over the last two glacial-interglacial cycles. Geol. Soc. America, special paper, 450, 169-196.

DEAN, W.E. et L.A. DONER (2011). Precipitation, Isotopic composition of endogenic carbonate, and vegetation history in a lake-Fen complex in northwestern Minnesota, open -File Report -1106. U.S. Geological Survey, Reston, Virginia.

DEAN, W.E., B. NEFF, D. ROSENBERRY, T.C. WINTER et R. PARKHURST (2003). The significance of ground water to the accumulation of iron and manganese in the sediments of two hydrologically distinct lakes in northcentral Minnesota - A geological perspective. Ground Water, 441, 951-963.

DEAN, W.E. et A. SCHWALB (2002). The lacustrine carbon cycle as illuminated by the waters and sediments of two hydrologically distinct hardwater lakes in northwestern Minnesota. J. Sedimentary Res., 72, 416-431.

DELIAN, F., L. TIEBING et Y. JIE (1992). The process of formation of manganese carbonate deposits hosted in black shale series. Econ. Geol., 87, 1419-1429.

DUAN, W.M., D.B. HEDRICK, K. PYE, M.L. COLEMAN et D.C. WHITE (1996). A preliminary study of the geochemical and microbiological characteristics of modern sedimentary concretions. Limol. Oceanogr., 41,1404-1414.

EFFLER, S.W. (1984). Carbonate equilibria and the distribution of inorganic carbon in Saginan Bay. J. Great Lakes Res., 10, 3-14. 
EFFLER, S.W. et D.L. JOHNSON (2007). Calcium carbonate precipitation and turbidity measurements in Otisco Lake, New York. J. Am. Water Resour. Assoc., 23, 73-79.

EHRENREICH, A. et F. WIDDEL (1994). Anaerobic oxidation of ferrous iron by purple bacteria, a new-type of phototrophic metabolism. Appl. Environ. Microb., 60, 4537-4526.

ELFIL, H. et H. ROQUES (2001). Role of hydrate phases of calcium carbonate on the scaling phenomenon. Desalination, 137, 177.

EMERSON, S. (1976). Early diagenesis in anaerobic lake sediments: Chemical equilibria in interstitial waters. Geochim. Cosmochim. Acta, 40, 925-934.

EUGSTER, H.P. et L.A. HARDIE (1978). Saline lakes, Dans : Lakes : Chemistry, geology, Physics. A. LEMAN (Editeur), Springer-Verlag, New York, pp. 237-294.

FORCE, E.R. et J.B. MAYNARD (1991). Manganese : Syngenetic deposits on the margin of anoxic basins. Rev. Econ. Geol., 5, 147-157.

FRAKES, L.A. et B.R. BOLTON (1992). Effects of Ocean Chemistry, sea level, and climate on the formation of primary sedimentary manganese ore deposits. Econ. Geol., 87, 1207-1217.

FREDERICHS, T., T. V. DOBENECK, U. BLEIL et M.J. DEKKERS (2003). Towards the identification of siderite rhodochrosite and vivianite in sediments by their lowtemperature magnetic properties. Phys. Chem. Earth, 28, 669-679.

GAL, J.Y., J.C. BOLLINGER, H. TOLOSA, N. GACHE (1996). Calcium carbonate solubility: a reappraisal of scale formation and inhibition. Talanta, 43, 1497-1509.

GAL, J.Y., Y. FOVET et N. GACHE (2002). Mechanisms of scale formation and carbon dioxide partial pressure influence. Part II. Application in the study of mineral waters of reference. Water Res., 36, 764-73.

GLASBY, G.P. et H.D. SCHULTZ (1999). EH, pH diagrams for $\mathrm{Mn}, \mathrm{Fe}, \mathrm{Co}, \mathrm{Ni}, \mathrm{Cu}$ and $\mathrm{As}$ under sea-water conditions: applications of two new types of $\mathrm{EH}, \mathrm{pH}$ diagrams to the study of specific problems in marine geochemistry. Aquat. Geochem, 5, 227-248.

GLENN, M.M. (2008). Eocene age fossilized filamentous bacteria: New evidence suggesting a bacterial genesis of siderite in the Green River formation, Wyoming. 28th Oil shale symposium Colorado School of Mines, $7 \mathrm{p}$.
GREEN, J.W., B.R. STAGE, B.J. BRATINA, S. WAGERS, A. PRESTON, K. O'BRYAN, J. SHACAT et S. NEWELL (2004). Nickel, copper, zinc and cadmium cycling with manganese in Lake Vanda (Wright valley, Antarctica). Aquat. Geochem., 10, 303-323.

GREINERT J., G. BOHRMANN et E. SUESS (2001). Gas hydrate-associated carbonates and methane-venting at hydrate Ridge: classification, distribution, and origin of authigenic lithologies. Dans: Natural gas hydrates: Occurrence, Distribution and Detection. PAULL, C.K. et W.P. DILLON (Editeurs), Geophys. Monogr. Ser., 124, 99-113, AGU, Washington, D.C.

HALBWACHS, M., J.C. SABROUX, J. GRANGEON, G. KAYSER, J.C TOUCHON-DANGUY, A. FELIX, J.C. BEARD, A. VILLEVIEILLE, G. VITTER, P. RICHON, A. WÜEST et J. HELL (2004). Degassing the "killer lakes " Nyos and Monoun, Cameroon. EOS Trans. AGU85, 281285.

HOUSE, W.A. (1984). The kinetics of calcite precipitation and related processes. Freshwater Biol. Assoc. Annu. Rep., Ambleside, 52, 75-90.

HSÜ, K.J. et K. KELTS (1978). Late Neogene sedimentation in the Black sea. Dans: Modern and Ancient Lake sediments. MATTER, A. et M.E. TUCKER (Editeurs), Oxford (Blackwell Scientific), IAS Spec. Publ., pp.129-145.

INTERNATIONAL REFERENCE GROUP (1977). Atmospheric loadings of the Lower Great Lakes and the Great Lake drainage basin. The International Reference Group on Great Lakes pollution from Land Use Activities, 79 p.

JENKYNS, H.C., B. GECZY et J.D. MARSHALL (1991). Jurassic manganese carbonates of Central Europe and the early Toarcien event. J. Geol., 137-149.

JIMENEZ-LOPEZ, C. et C.S. ROMANEK (2004). Precipitation kinetics and carbon partitioning of inorganic siderite at $25^{\circ} \mathrm{C}$ and $1 \mathrm{~atm}$. Geochim. Cosmochim. Acta, 68, 557-571.

JOHANESS, W. et D. PUHAN (1971) The calcite-aragonite transition, reinvestigated. Mineral. Petrol., 31, 28-38.

JOHNSON, M.L. (1990). Ferrous carbonate precipitation kinetics. A temperature ramped approach. PhD. Rice University, Houston, Texas, 139 p.

JOHNSON, J.W., E.H. OELKERS et H.C. HELGESON (1992). SUPCRT92: A software package for calculating the standard molal thermodynamic properties of minerals, 
gases, aqueous species, and reactions from 1 to 5000 bars and 0 to $1000^{\circ} \mathrm{C}$ : Computers \& Geosciences, 18, 899-947.

KAMGAING, T. (2003 a). Étude comparée du caractère des eaux de deux lacs de cratère à risque majeur pour l'environnement : le Lac Nyos et le Lac Monoun OuestCameroun. Tribune de l'eau, 56, 52-59.

KAMGAING, T. (2003 b). Caractère agressif des eaux du Lac Nyos. Impact du dégazage de l'hypolimnion et suggestions. Tech. Sci. Meth.(TSM), 10, 59-65.

KAPPLER, A. et D.K. NEWMAN (2004). Formation of Fe3+minerals by Fe2+-oxidizing photoautotrophic bacteria. Geochim. Cosmochim. Acta, 68, 1217-1226.

KAPPLER, A., K.L. STRAUB (2005). Geomicrobiological cycling of iron. Dans: Molecular Geomicrobiology. BANFIELD, J.F., J. CERVINI-SILVA et K.H. NEALSON (Editeurs), 59, pp. 85-108.

KAWASHIMA, M., T. TAKAMATSY et M. KOYAMA (1988). Mechanisms of precipitation of manganese (II) in Lake Biwa, a fresh water lake. Water Res., 22, 613-618.

KELTS, K. et K.J. HSÜ (1978). Freshwater carbonate sedimentation. In: Lakes - chemistry, geology, physics. LERMAN A. (Editeur), Springer, pp. 295-323.

KHIM, B.K., K.S. CHOI, Y.A. PARK et J.K. OH (1999). Occurrence of authigenic siderites in the early Holocene Coastal deposit in the west coast of Korea: an indicator of depositional environment. Geosci. J., 3,163-170.

KLING, G.W., M.A. CLARK, H.R. COMPTON, J.D. DEVINE, W.C. EVANS, A.M. HUMPHREY, E.J. KOENISBERG, J.P. LOCKWOOD, M.L. TUTTLE et G.N. WAGNER (1987). The 1986 Lake Nyos gaz disaster in Cameroon, West-Africa. Science, 236, 169-175.

KLING, G.W., W.C. EVANS, G. TANYILEKE, M. KUSAKABE, T.OHBA, Y. YOSHIDA et J.V. HELL (2005). Degassing Lakes Nyos and Monoun: defusing certain disaster. Proc. Natl. Acad. Sci USA, 102, 1418514190.

KONHAUSER, K.O. (1998). Diversity of bacterial iron mineralization. Earth Sci. Rev., 43, 91-121.

KONOVALOV, S., A. SAMODUROV, T. OGUZ et L. IVANOV (2004) Parameterization of iron and manganese cycling in the Black Sea suboxic and anoxic environment. Deep-Sea Research I, 21, 2027-2045.
KRYLOV, A., O. KHLYSTOV, T. ZEMSKAYA, H. MINAMI,

A. HACHIKUBO,Y. NUNOKAWA, M. KIDA, H. SHOJI, L. NAUDTS, J. POORT et T. POGODAEVA (2008). First discovery and formation process of authigenic siderite from gas-hydrate-bearing mud volcanoes in fresh water: Lake Baikal, eastern Siberia. Geophys. Res. Lett., 35, L05405 (6 p.).

KULIK, D.A., M. KERSTEN, U. HEISER et T. NEUMANN (2000). Application of Gibbs energy minimization to model early-diagenetic solid-solution aqueous-solution equilibria involving authigenic rhodochrosites in anoxic Baltic Sea sediments. Aquat. Geochem., 6, 147-199.

KUSAKABE, M., G.Z.TANYILEKE, S.A. McCORD et S.G. SCHLADOW (2000). Recent $\mathrm{pH}$ and CO2 profiles at Lakes Nyos and Monoun, Cameroon: implications for the degassing strategy and its numerical simulation. J.Volcanol. Geotherm.Res., 97, 241-260.

KUSAKABE, M., T. OHBA, ISSA, Y. YOSHIDA, H. SATAKE, T. OHIZUMI, W.C. EVANS, G. TANYILEKE et G.W. KLING (2008). Evolution of CO2 in Lakes Monoun and Nyos, Cameroon, before and during controlled degassing. Geochem. J. 42, 93-118.

LANGMUIR, D. (1997) Aqueous Environmental Geochemistry. Prentice Hall, Englewood Cliffs, New Jersey, 600 p.

LAST, M.W. et P. DE DECKKER (1990). Modern and Holocene carbonate sedimentology of two saline volcanic maar lakes, Southern Australia. Sedimentology, 37, 967981.

LEBRON, I. et D.L. SUAREZ (1996). Calcite nucleation and precipitation kinetics as affected by dissolved organic matter at $25^{\circ} \mathrm{C}$ and $\mathrm{pH}>7.5$. Geochim. Cosmochim. Acta, 60, 2765-2776.

LEGRAND, L. et G. POIRIER (1976). Chimie des eaux naturelles, Eyrolles, Paris, 312 p.

LEIN, A.Y. (2004). Authigenic carbonate formation in the Ocean. Lithol. Miner. Resour. 39, 1-30.

LEROY, P. (1994). Le plomb dans l'eau: origine et influence des caractéristiques des eaux transportées. Tech. Sci. Meth. (TSM), 3, 122-127.

LEWIS, B.L. et W.M. LANDING (1991). The biogeochemistry of manganese and iron in the Black Sea. Deep Sea Res., 38(suppl. À), S773-S804. 
LIM, D.I., H.S. JUNG, S.Y. YANG et H.S. YOO (2004). Sequential growth of early diagenetic freshwater siderites in the Holocene coastal deposition, Korea. Sediment. Geol., 169, 107-120.

LOVLEY, D.R. (1993). Dissimilatory Metal Reduction. Ann. Rev. of Microbiol., 47, 263-290.

LOVLEY, D.R. (2004) Potential role of dissimilatory iron reduction in the early evolution of microbial respiration. Dans: Origins, Evolution and Biodiversity of Microbial life. SECKBACH J. (Editeur), pp. 299-313.

MASON, L.M., A.R.HARRIS, C.M. BIRKETT, W.CUDLIP et C.E. RAPLEY (1991). Remote sensing of lakes for the proxy monitoring of climatic change. Proc. 16th Ann. Conf. Remote Sensing Society, pp. 314-324.

MAYHEW, S.G. (1978). The redox potential of dithionite and SO2- from Equilibrium reactions with flavodoxins, methyl viologen and hydrogen plus hydrogenase. Eur. J. Biochem, $85,535-547$.

MIZANDRONTSEV, I.B. (1975). About geochemistry of the pore solutions (in Russian). Dans: Dynamic of the Baikal Depression. GALAZii G.I. et Y.P. PARMUZIN (Editeurs), Nanka, Novosibirsk, Russia, pp. 203-231.

MORAD S. et I.S. Al-AaSM (1997). Conditions of Rhodochrosite-nodule formation in Neogene-Pleistocene deep-sea sediments: Evidence from $\mathrm{O}, \mathrm{C}$ and $\mathrm{Sr}$ isotopes. Sediment. Geol., 114, 295-304.

MORSE, W. J., S.R. ARVIDSON et A. LÜTTGE (2007). Calcium carbonate formation and dissolution. Chem. Rev. 107, 342-381.

MORTIMER, R.J.G. et M.L. COLEMAN (1997). Microbial influence on the oxygen isotopic composition of diagenetic siderite. Geochim. Cosmochim. Acta, 61, 1705-1711.

MOORE, S.E., J.R.E. FERRELL et P. AHARON (1992). Diagenetic siderite and other ferroan carbonates in a modern subsiding marsh sequence. J. Sediment. Petrol., 62, 357-366.

MOZLEY, P.S. et W.W. CAROTHERS (1992). Elemental and isotopic composition of siderite in the Kupanuk Formation, Alaska: effect of microbial activity and water/ sediment interaction on early pore-water chemistry. $J$. Sediment. Petrol., 64, 681-692.

MÜLLER, B., Y. WANG et B. WEHRLI (2006). Cycling of calcite in hard water lakes of different trophic states. Limnol. Oceanogr., 51, 1678-1688.
MÜLLER, G., IRION et U. FÖRSTNER (1972). Formation and diagenesis of inorganic $\mathrm{Ca}-\mathrm{Mg}$ carbonates in the lacustrine environment. Naturewissenschaften, 59, 158164.

MUNCH, J.C. et J.C.G. OTTOW (2010). Réduction bactérienne des oxydes ferriques amorphes et cristallisés. Association Française pour l'Étude du Sol, 205-215.

MURPHY, T.P., K.J. HALL et I. YESAKI (1983). Coprecipitation of phosphate with calcite in a naturally eutrophic lake . Limnol. Oceanogr., 28, 58-69.

MURRAY, J.W., L.A. CODISPOTI et G.E. FRIEDERICH (1995). Oxidation-reduction environments. The suboxic zone in the Black Sea. Dans: Aquatic Chemistry: Interfacial and Interspecies Processes. HUANG, C.P., C.R. O'MELIA, J.J. MORGAN (Editeurs), ACS Advances in Chemistry Series, 244, pp.157-176.

NAN, Z., Z. SHI, M. QIN, W. HOU et Z. TAN (2007). Formation process and Thermodynamic properties of calcite. Chin. J. Chem. 25, 592-595.

NEALSON, K.H.et D. SAFFARINI (1994). Iron and manganese in anaerobic respiration, environmental significance, physiology and regulation. Annual Review of Microbiology, 48, 311-343.

NIA P., V.B.K. KAMGANG, L.N. SIGHA, C.W. EVANS et G. TANYILEKE (2009). Fonctionnement hydrogéochimique du Lac Nyos avant son dégazage. Syllabus Review, 1, 16-27.

NEUMANN, T., C. CHRISTIANSEN, S. CLASEN, K.C. EMERIS et H. KUNZENDORF (1997). Geochemical records of salt water inflows into the deep basins of the Baltic Sea. Cont. Shelf Res., 17, 95-115.

NEUMANN, T., U. HEISER, M.A. LEOSSON et M. KERSTEN (2002). Early diagenetic processes during Mn-carbonate formation: evidence from the isotopic composition of authigenic Ca-rhodochrosites of the Baltic Sea. Geochim. Cosmochim. Acta, 66, 867-879.

NEVIN, K.P. et D.R. LOVLEY (2002). Mechanisms for $\mathrm{Fe} 3+$ oxide reduction in sedimentary environments. Geomicrobiol. J., 19, 141-159.

OGUZ, T., J.W. MURRAY et A. CALLAHAN (2001). Modeling redox cycling across the suboxic-anoxic interface zone in the Black Sea. Deep-Sea Res., 48, 761-787.

OKITA, P.M. et W.C. SHANKS (1992). Origin of stratiform sediment-hosted manganese carbonate ore deposits: 
examples from Molango, Mexico and Tao Tiang, China. Chem. Geol., 99,139-164.

PEARSON, M.J. (1979). Geochemistry of the Hepworth carboniferous sediment sequence and the origin of the diagenetic iron minerals and concretions. Geochim. Cosmochim. Acta, 43, 927-941.

PIENITZ, R., I.R. WALTER et B.A. ZCEB (1992). Biomonitoring past salinity changes in an athalassic subartic lake. Int. J. salt lake Res., 1, 91-123.

PISAPIA, C., M. CHAUSSIDON, C. MUSTIN et B. HUMBERT (2007). O and $S$ isotopic composition of dissolved and attached oxidation products of pyrite by Acidithiobacillus ferroxidans : comparison with abiotic oxidations. Geochim. Cosmochim. Acta, 71, 2474-2490.

PLUMMER, L.N., T.M.L. WIGLEY et D.L. PARKHURST (1978). The kinetics of calcite dissolution in CO2-water systems at 5-60 ${ }^{\circ} \mathrm{C}$ and 0.0-1.0 atm. CO2. Am. J. Sci., 278, 179-216.

POURBAIX, M. (1963). Atlas d'Equilibres Electrochimiques à $25^{\circ} \mathrm{C}$. Gauthier-Villars, Paris, $644 \mathrm{p}$.

POSTMA, D. (1982). Pyrite and siderite formation on brackish and freshwater swamp sediments. Am. J. Sci., 282, 1151-1183.

PYE, K. (1984). SEM analysis of siderite cements in intertidal marsh sediments, Norfolk, England. Mar. Geol., 56, 1-12.

QUERALT, I., R. JULIA, F. PLANA et J.L. BISCHOFF (1997). A hydrous Ca-bearing magnesium carbonate from Playa lake sediments, Saline Lakes, Spain. Am. Mineral., 82, 812-819.

RAJAN, S., F. MACKENZIE et C.R. GLENN (1996). A thermodynamic model for water column precipitation of siderite in the plio-pleistocene Black sea. Am. J. Sci., 296, 506-548.

ROH, Y., C.L. ZHANG, H. VALI, R.J. LAUF, J. ZHOU et T.J. PHELPS (2003). Biogeochemical and environmental factors in Fe biomineralization : Magnetite and siderite formation. Clays Clay Miner. 51, 83-95.

ROH, Y.,H. GAO, H. VALI, D.W. KENNEDY, Z.K. YANG, W. GAO, A.C. DOHNALKOVA, R.D. STAPLETON, J.W. MOON, T.J. PHELPS, J.K. FREDRICKSON et J. ZHOU (2006). Metal reduction and iron biomineralization by a psychrotolerant $\mathrm{Fe}(\mathrm{III})$-Reducing Bacterium shewanella sp. Strain PV-4. Appl. Environ. Microbiol.,72, 3236-3244.
ROCA, J.R. et R. JULIA (1997). Late-Glacial and Holocene lacustrine sequence evolution based on ostracode assemblages in south-easten Spain. Geobios., 30, 823-830.

RODO, X., A. F. COMIN, G. R. TENORIO et R. JULIA (2003). High-resolution saline lake sediments as enhanced tools for relating proxy paleolake records to recent climatic data series. Sedimentol. Geol., 148, 203-220.

ROELS, J. et W. VERSTRAETE (2001). Biological formation of volatile phosphorus compounds. Biosci. Technol., 79, 243-250.

ROMANEK, C.S., C.L. ZHANG, Y. LI, H. VALI, J. HORITA et D.R COLE (2003). Carbon and hydrogen isotope fractionations associated with dissimilatory iron-reducing bacteria. Chem. Geol. (Isot. Geosci. Sect.) 195, 5-16.

ROMANEK, C.S., C. JIMENEZ-LOPEZ, R.A. NAVARRO, M. SANCHEZ ROMAN, N. SAHAI et M. COLEMAN (2009). Inorganic synthesis of Fe-Ca-Mg carbonates at low temperature. Geochim. Cosmochim. Acta, 73, 5361-5376.

ROSSMANN, R. (1980). Inorganic chemistry of particulate matter from the nearshore zone of Lake Michigan. J. Great Lakes Res., Int. Assoc. Great Lakes Res., 6, 348-352.

SAPOTA, T., A. ALDAHAN et I.S. AL-AASM (2006). Sedimentary facies and climate control on formation of vivianite and siderite microconcretions in sediments of Lake Baikal, Siberia. J. Paleolimnol., 36, 245-257.

SCHMID, M., M. HALBWACHS et A. WÜEST (2006). Simulation of $\mathrm{CO} 2$ concentrations, temperature and stratification in Lake Nyos for different degassing scenarios. Geochem. Geophys. Geosyst., 7, Q06019.

SHAOFENG, J., K. DO-GUN, K. JEONGHYUN et K. SEOK-OH (2010). Characterization of the biogenic Manganese oxides produced by pseudomonas putida Strain MnB1. Environ. Eng. Res. 15, 183-190.

SHAPLEY, M., E. ITO, J. DONOVAN et D. ENGSTROM (2002). Endogenic carbonate sediment flux in lakes as indicator of Paleo-Groundwater recharge. American Geophys. Union, Fall Meeting, Abstract PP51A-0298.

SHOW, N., S. MORAD et I.S. AL-AASM (2000). Origin of authigenic Mn-Fe carbonates and pore-water evolution in marine sediments: Evidence from Cenozoic strata of the Arctic Ocean and Norwegian-Greenland Sea (ODP Leg 151). J. Sediment. Res., 70, 682-699.

SIGURDSSON, H., J.D. DEVINE, F.M. TCHOUA, T.S. PRESSER, M.K.W. PRINGLE et W.C. EVANS (1987). 
Origin of the lethal burst from Lake Monoun, Cameroon. J. Volcanol. Geotherm. Res., 31,1-16.

SINGER, P.C. et W. STUMM (1970) The solubility of ferrous iron in carbonate-bearing waters. J. Am. Water Works Assoc., 62, 198-202.

STABEL, H.H. (1986). Calcite precipitation in Lake Constance: Chemical equilibrium, sedimentation, and nucleation by algae. Limnol. Oceanogr., 31, 1081-1093.

STRAUB, K.L., M. BENZ, B. SCHINK et F. WIDDEL (1996). Anaerobic, nitrate-dependent microbial oxidation of ferrous iron. Appl. and Environ. Microb., 62, 1458-1460.

SCHELOBOLINA, E.S., C.G. VANPRAAGH et D.R. LOVLEY (2003). Use of ferric and ferrous iron containing minerals for respiration by Desulfitobacterium frappieri. Geomicrobiol. J., 20, 143-156.

STEVENS, L.R., E. ITO et D.E.L. OLSON (2000). Relationship of Mn-carbonates in varved lake sediments to catchment vegetation in Big Watab Lake, MN, USA. J. Paleolim., 24,199-211.

STRAUB, K.L. et B.E.E BUCHHOLZ-CLEVEN (1998). Enumeration and detection of anaerobic ferrous ironoxidizing nitrate-reducing bacteria from diverse European sediments. Appl. Environ. Microb., 64, 4846-4856.

STUCKI. J.W. (2006). Iron redox processes in clay minerals. Dans: Handbook ofClay Science. BERGAYA. F., G. LAGALY et B.G.K. THENG (Editeurs), Elsevier, Amsterdam.

STUMM. W. et J.J. MORGAN (1996). Aquatic Chemistry, Chemical Equilibria and Rates in Natural Waters, 3rd edition. John WILLY\& Sons, Inc., New York, 1022 p.

TEBO, B.M. (1991). Manganese(II) oxidation in the suboxic zone of the Black sea. Deep-Sea Research, 38 (Suppl. 2A)), S883-S906.

TELLER, J.T. et W.M. LAST (1990). Paleohydrological indicators in Playas and salt lakes, with examples from Canada, Australia and Africa. Palaeogeogr., Palaeoclimatol. Palaeoecol., 76, 215-240.

TEUTSCH, N., M. SCHMID, B. MÜLLER, A.N. HALLIDAY, H. BÜRGMANN et B. WEHRLI (2009). Large iron isotope fractionation at the oxic-anoxic boundary in Lake Nyos. Earth Planet. Sci. Lett. 285, 52-60.

UPPER LAKES REFERENCE GROUP (1976). The water of Lake Huron and Lake Superior, Vol. I, Summary and
Recommendations: Report to the International Joint Commission, 236 p.

WERSIN, P., L. CHARLET, R. KARTHEIN et W. STUMM (1989). From adsorption to precipitation: Sorption of Mn2+ on FeCO3(s). Geochim. Cosmochim. Acta, 53, $2787-$ 2796.

WHITICAR, M.J. (1999). Carbon and hydrogen isotope systematic of bacterial formation and oxidation of methane. Chem. Geol., 161, 291-314.

WIDDEL, F., S. SCHNELL, S. HEISING, A. EHRENREICH, B. ASSMUS et B. SCHINK (1993). Ferrous iron oxidation by anoxygenic phototrophic bacteria. Nature, 362, 834836.

ZHANG, C.L., H. VALI, C.S. ROMANEK, Y. ROH, S.K. SEARS et T.J. PHELPS (1998). Chemical and morphological characterization of siderite formed by iron reducing bacteria. Am. Mineral., 61,927-932.

ZHANG, C.L., J. HORITA, D.R. COLE, J. ZHOU, D.R. LOVLEY et T.J. PHELPS (2001). Temperature-dependent oxygen and carbon isotope fractionation of biogenic siderite. Geochim. Cosmochim. Acta, 65, 2257-2271.

ZHANG, G., H. DONG, H. JIANG, K.R. KUKKADAPU, J. KIM, D. EBERI et Z. XU (2009). Biomineralization associated with microbial reduction of $\mathrm{Fe}^{3+}$ and oxidation of $\mathrm{Fe}^{2+}$ in solid minerals. Am. Mineral., 94, 1049-1058.

ZHAODONG, N., C. XIANGNA, Y. QIANQIAN, W. XIUZHEN et H. WANGUO (2008). Structure transition from aragonite to vaterite and calcite by the assistance of SDBS. J. Coll. I. Sc., 325, 331-336.

ZODROW, E.L., P.C. LYONS et M.A. MILLWAY (1996). Geochemistry of autochthonous and hypautochthonous siderite-dolimite coalballs (Foord Seam, Bolsovian, upper carboniferous), Nova Scotia, Canada. Int. J. Coal Geol., 29, 199-216. 